Sen and Bhakat/ Journal of Tropical Forestry and Environment Vol. 10 No. 01 (2020) 17-39

\title{
Floristic and Phytoclimatic Study of an Indigenous Small Scale Natural Landscape Vegetation of Jhargram District, West Bengal, India
}

\author{
U.K. Sen* and R.K. Bhakat \\ Ecology and Taxonomy Laboratory, Department of Botany and Forestry, Vidyasagar University, \\ West Bengal, India
}

Date Accepted: 28-06-2020

\begin{abstract}
Sacred groves are distinctive examples of biotic components as genetic resources being preserved in situ and serve as secure heavens for many endangered and endemic taxa. From this point of view, the biological spectrum, leaf spectrum and conservation status of the current sacred grove vegetation, SBT (Swarga Bauri Than) in Jhargram district of West Bengal, India, have been studied. The area's floristic study revealed that SBT's angiosperms were varied and consisted of 307 species belonging to 249 genera, distributed under 79 families of 36 orders as per APG IV. Fabales (12.05\%) and Fabaceae (11.73\%) are the dominant order and family in terms of species wealth. Biological spectrum indicates that the region enjoys "thero-chamae-cryptophytic" type of phytoclimate. With respect to the spectrum of the leaf size, mesophyll (14.05\%) was found to be high followed by notophyll (7.84\%), microphyll (7.19\%), macrophyll (7.84\%), nanophyll (6.86\%), leptophyll (6.21\%), and megaphyll (2.29\%). The study area, being a sacred grove, it has a comparatively undisturbed status, and the protection of germplasm in the grove is based on traditional belief in the social system.
\end{abstract}

Keywords: biodiversity conservation, biological spectrum, leaf spectra, life-form, sacred grove

\section{Introduction}

Sacred groves are indigenous small scale natural landscape of native vegetation kinds that are traditionally protected and managed by local populations. A range of taboos and prohibitions are used to preserve biodiversity in 'sacred groves' (Colding and Folke, 1997, 2001; Berkes, 2009). Many of them are connected to the premises of tiny temples. These sacred groves comprise plant species that are endemic, rare and endangered. They are therefore natural nursery with rare, threatened and endemic plant species, many of which have vanished outside the groves from the region (Colding and Folke, 1997).

Local communities preserve and protect sacred groves because of their religious convictions and the related traditional rituals that run through several generations. They may consist of multi-species, multitier primary forests or a clump of trees, depending on the history of the vegetation (Gokhale et al., 2011). According to Hughes and Chandran (1998), these groves are landscape segments comprising vegetation and other types of life and geographical characteristics that are delimited and protected by human communities, believing that maintaining them in a comparatively undisturbed state is an expression of human relationship with the divine or nature. Such groves are often situated in biodiversityrich areas, ranging from a few trees to multi-acre forestland. Adapting a plant to certain ecological circumstances determines a type of life; therefore, it is a significant feature of physiognomy that has been commonly used in vegetation assessment. It shows the macro and microclimate and human disturbances of a certain area (Cain and Castro, 1959).

*Correspondence: uudaysen@gmail.com

Tel: +919002524806

(C) University of Sri Jayewardenepura 
The word "biological spectrum" was suggested by Raunkiaer (1934) to describe the distribution of life-form in a flora as well as the phytoclimate under which the prevailing life-forms developed.Under this scheme, plant species can be divided into five primary groups, i.e., phanerophytes, chamaephytes, hemicryptophytes, cryptophytes and therophytes.

The percentage of various life form classes put together is called as the biological spectrum. Raunkiaer (1934) built a standard spectrum that could behave as a null model, compared to different spectra of life form. The standard spectrum of Raunkiaer (1934) shows a phanerophytic community, and the deviation (from it) determines the habit's phytoclimate. The occurrence in separate areas of comparable biological spectra shows comparable climatic circumstances. Thus, the differences between normal spectrum and biological spectrum life forms may point out which life form characterises the phytoclimate or vegetation.

Climatic types may be characterised by the prevailing plant life forms in the plant communities under a specific climatic regime (Raunkiaer, 1934; Cain, 1950; Muller and Ellenberg, 1974; Saxena et al., 1982). The Indian region's biological spectrum is linked to particular edaphic, altitude and climatic variables (Meher-Homji, 1964; Rana et al., 2002; Reddy et al., 2011; Sen and Bhakat, 2009, 2012; Singh and Gupta, 2015; Sen, 2016, 2018; Sen and Bhakat, 2018, 2019a, b, c). Studying life-form is therefore a significant component of the description of vegetation, ranking next to floristic structure (Batalha and Martins, 2004). Therefore, the biological spectrum is helpful as an index of forest landscape health status. Biological spectrum may set rules for a community's optimisation and eco-restoration when performed at regular intervals.

Life form may also be categorised using leaf size i.e., leptophylls, nanophyll, microphyll, notophyll, mesophyll, macrophyll and megaphyll. It has some justification for using a leaf size to characterise distinct kinds of vegetation based on percentages of the distinct leaf dimensions present. However, light intensity and soil conditions, especially nitrogen and phosphorus accessible; also have a significant impact on the size of the leaf even within the same genotype (Cunningham et al., 1999).

\section{Materials and Methods}

2.1 Study site

a) The sacred grove

The study was conducted in a forested sacred grove namely SBT on outer edge of a tribal dominated Chhotopindara, Ranijhor and Dochakhuria villages along the south-western bank of a perennial rivulet Palpala, under Gidni block (latitude $22^{\circ} 26^{\prime} 00.09^{\prime \prime}-22^{\circ} 26^{\prime} 01.48^{\prime \prime} \mathrm{N}$ and longitude $86^{\circ} 50^{\prime} 00.90^{\prime \prime}-86^{\circ} 50^{\prime} 01.56^{\prime \prime} \mathrm{E}$, average altitude $86.7 \mathrm{~m}$ asl) in Jhargram district of West Bengal, India (Figure. 1, 2, 3). The grove houses a brick-made small temple and is spread over a 3.5 acre public land. The grove is located about $38 \mathrm{~km}$ southeast from district headquarters at Jhargram town, located in the southern part of West Bengal, India (Figure. 1).

It represents a 400-450-year-old relict forest patch consisting of evergreen, deciduous and semideciduous plants. After the eight days of annual Paus Sankranti (a ritual celebrated on the last day of the Bengali Month Paus or middle of January) and every Tuesday and Saturday local people, both tribal and non-tribal of Gidni and adjoining blocks, visit the grove and worship the deity. Since the grove is an abode of deity, the entire area along with plants and other life forms is considered sacred. Owing to this socio-cultural tag on the grove, local people do not cut or disturb the grove flora, thus strictly adhering to the taboos and ethics. 
Jhargram district covers an area of 3,037.64 $\mathrm{km}^{2}$ and had a population of 1,136,548 in the 2011 census. $96.52 \%$ of the total population were rural and only $3.48 \%$ were urban population. $20.11 \%$ of the total population belonged to scheduled castes and $29.37 \%$ belonged to scheduled tribes. Its population growth rate over the decade 2001-2011 was $10.9 \%$. The literacy rate was $72 \%$ in 2011 , where the male literacy rate was $81 \%$ and female at $64 \%$. The sex ratio was 979 females per 1,000 males (Anon, 2011).

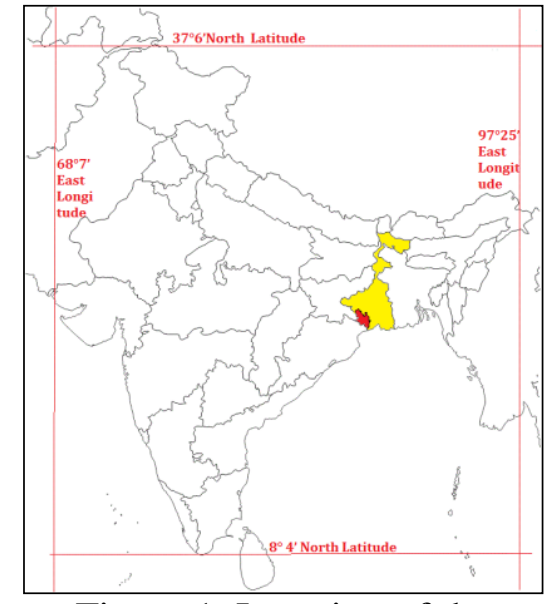

Figure 1. Location of the study area.

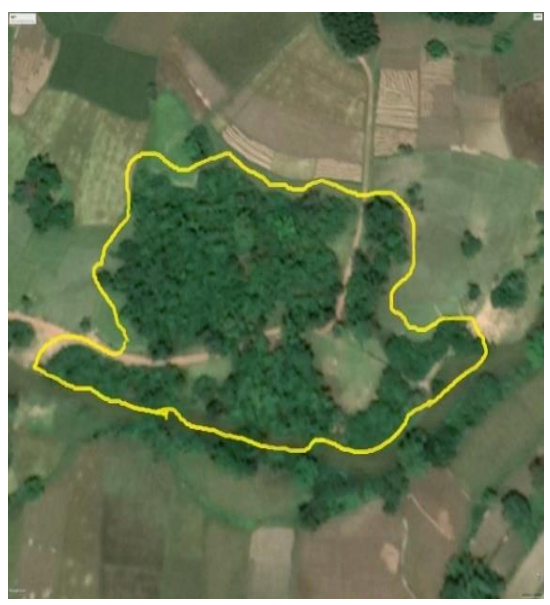

Figure 2. Google earth image showing SBT

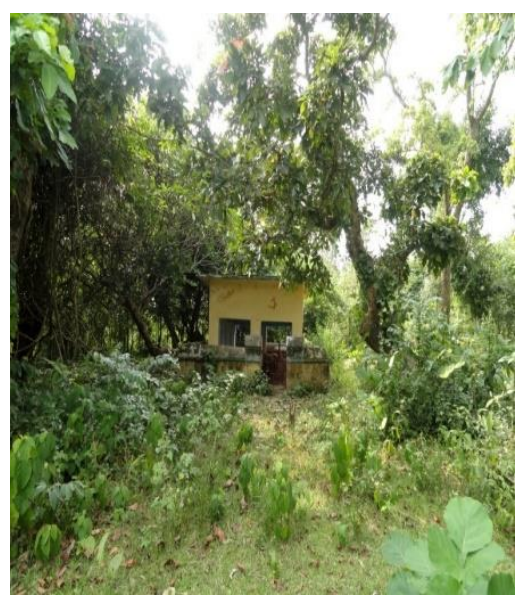

Figure 3. Small temple in SBT sacred grove.

\subsection{Field survey and data collection}

During the period from December 2012 to January 2019, the study area was carefully surveyed in various seasons to explore the botanical and social wealth. A short floristic study was conducted on the grounds of "spot identification". Samples of plants with flowers or fruits have been gathered for unknown plants. The samples were processed, maintained, poisoned and assembled on herbarium sheets using conventional and modern herbarium methods after collection (Jain and Rao, 1977). In the sacred grove, photographs were taken of some prevalent, locally rare, endemic and valuable plant species. Herbarium sheets have been recognised by matching properly annotated materials available at Vidyasagar University's Herbarium. For identification purpose, different relevant catalogue (Anderson, 1862), regional floras (Hooker, 1872-1897; Prain, 1903; Haines, 1921-1925; Bennet, 1979; Sanyal, 1994), monographs (Mitra, 1958), revisionary works (Datta and Majumdar, 1966) and other literature were consulted. The socio-cultural functions surrounding the grove were recorded through information collected by interviewing and cross-interviewing the local people.

\subsection{Analysis of vegetation}

In the systematic enumeration of the taxa; clade, order, family, species along with habit, lifespan, flowering and fruiting time, Raunkiaer's life-form with sub-type, leaf spectra, IUCN status (IUCN, 2020) and distribution of the plants in the grove have been arranged according to Angiosperm Phylogeny Group IV classification (Chase et al., 2016) (Table 1). All the species were categorised into various Raunkiaer's life form categories depending on the position of regenerating parts or propagules in all the collected species. Thus a biological spectrum was prepared for the grove that was subsequently compared with the Raunkiaer's normal spectrum to determine the phytoclimate of the grove (Raunkiaer, 1934; Muller and Ellenberg, 1974). The knowledge of leaf size helped us understand the physiological status of plants and the plant communities were useful in classifying the associations of plants. Plants were divided into (a) leptophyll $\left(<25 \mathrm{~mm}^{2}\right)$, (b) nanophyll $\left(25-225 \mathrm{~mm}^{2}\right)$, (c) microphyll $(225-2,025$ 
$\left.\mathrm{mm}^{2}\right)$, (d) notophyll $\left(2,025-4,500 \mathrm{~mm}^{2}\right)$, (e) mesophyll $\left(4,500-18,225 \mathrm{~mm}^{2}\right)$, (f) macrophyll $(18,225-$ $\left.164,025 \mathrm{~mm}^{2}\right)$ and $(\mathrm{g})$ megaphyll $\left(>164,025 \mathrm{~mm}^{2}\right)$ (Raunkiaer, 1934).

\section{Results and Discussion}

\subsection{Different plant taxa}

In the present study, a total of 307 species belonging to 249 genera distributed over 79 families under 36 orders (APG IV, 2016) were recorded from the sacred grove. The top two clades are Rosids and Asterids. More than $81 \%$ of the flora is represented by orders of Eudicot and Core Eudicot, of which the major contributions in terms of descending species number ( $\geq 10$ species) are from Fabales 37 (12.05\%), Lamiales 36 (11.73\%), Gentianales 28 (9.12\%), Poales 28 (9.12\%), Malvales 20 (6.51\%), Asterales 17 (5.54\%), Malpighiales 17 (5.54\%), Myrtales 15 (4.89\%), Solanales 14 (4.56\%), Sapindales $13(4.23 \%)$ and Caryophyllales 12 (3.91\%) (Table 1, Figure. 4). Similar types of distribution of orders were highlighted by Gnanasekaran et al., 2012; Sen, 2016, 2018 and Sen and Bhakat, 2018, 2019a, b, c.

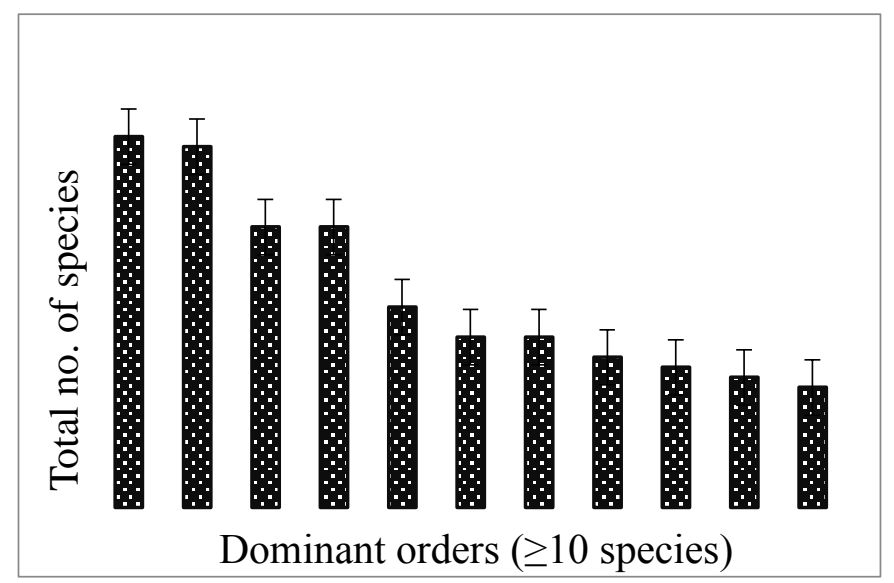

Figure 4. Major contribution of orders ( $\geq 10$ species) in the SBT.

The fourteen well represented families in species ( $\geq 6$ species), are: Fabaceae 36 (11.73\%), Poaceae 20 (6.51\%), Malvaceae 19 (6.19\%), Apocynaceae 18 (5.86\%), Asteraceae 17 (5.54\%), Lamiaceae 15 (4.89\%), Acanthaceae 12 (3.91\%), Euphorbiaceae 9 (2.93\%), Rubiaceae 9 (2.93\%), Cyperaceae 8 (2.61\%), Solanaceae 8 (2.61\%), Amaranthaceae 6 (1.95\%), Combretaceae $6(1.95 \%)$ and Convolvulaceae 6 (1.95\%) (Table 1, Figure. 5). Cucurbitaceae, Moraceae and Phyllanthaceae comprised $5(1.62 \%)$ species each. Four families contained $4(1.30 \%)$, eight families contained $3(0.98 \%)$ and thirteen families covered $2(0.65 \%)$ species. Another 37 families each had only a single species (Table 1).

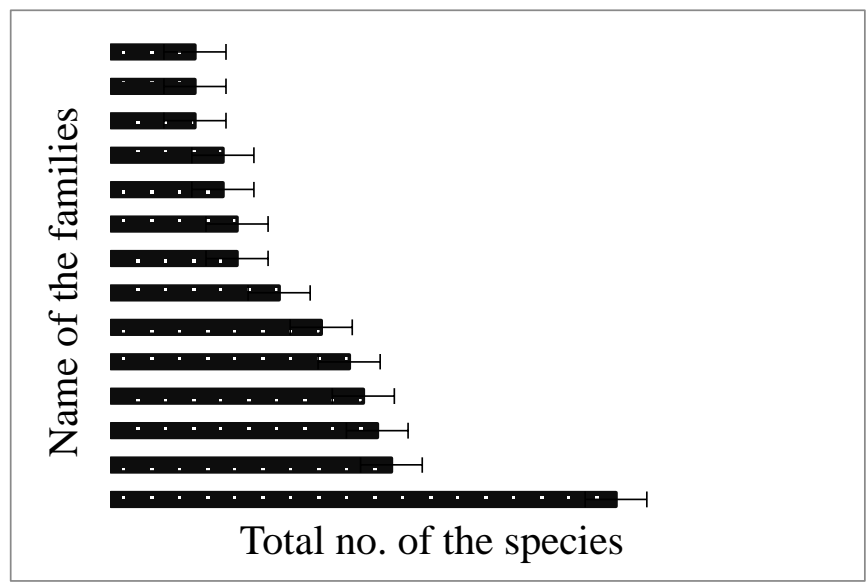

Figure 5. Major contribution of families ( $\geq 6$ species) in the SBT. 
Same type dominant families of sacred groves in India were observed by Rajendraprasad et al., 1998; Ghildiyal et al., 2016; Sen, 2016 etc. In the global context, such family dominance was shown by Batalha and Martins, 2004; Badshah et al., 2016; Chigani et al., 2017 etc. Asteraceae, Fabaceae and Poaceae emerged as the common families in the investigated area. Mendez (2005) also stated that the abundance of the same families in Laguna (Mendoza, Argentina). The members of Fabaceae and Poaceae were dominant due to their distribution with wide ecological amplitude.

\subsection{Species diversity in different growth forms}

The present floristic study of the sacred grove showed that it harboured a total of 307 plant species [dicots $249(81.11 \%)$ and monocots $58(18.89 \%)$ ] belonging to 249 genera [dicots $203(81.53 \%)$ and monocots $46(18.47 \%)$ ] of 79 families [dicots $62(78.48 \%)$ and monocots $17(21.52 \%)$ ] under 36 orders [dicots $27(75 \%)$ and monocots $9(25 \%)$ ]. Among these, 119 (38.76\%) of the reported species were herbs followed by shrubs $63(20.52 \%)$, trees $76(24.76 \%)$ and climbers 49 (15.96\%) respectively. Amongst the total dicots 249 (81.11\%) and monocots 58 (18.89\%), herbs, shrubs, trees and climbers represented 79, 59, 70, 41 and 40, 4, 6, 8 species respectively, representing 25.73\%, 19.22\%, 22.80\%, $13.36 \%$ and $13.03 \%, 1.30 \%, 1.95 \%, 2.61 \%$ of the total species (Table 2, Figure 6).

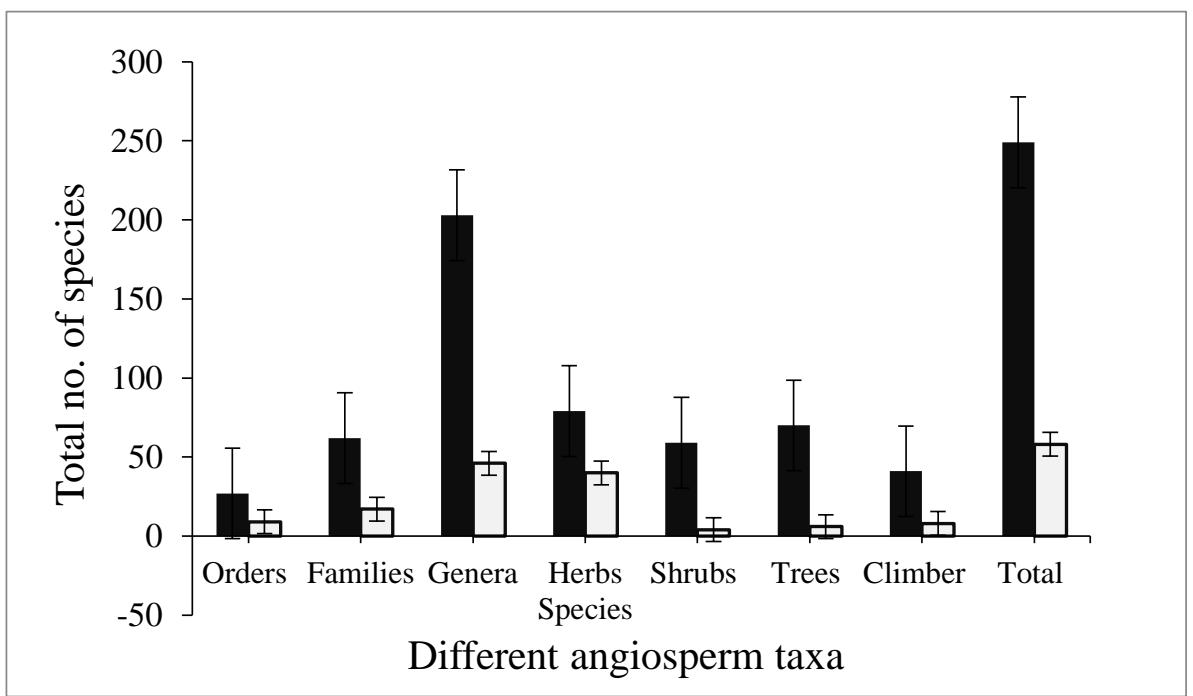

Figure 6. Total angiosperm taxa. 
Table 1: Floristic list of SBT sacred grove.

\begin{tabular}{|c|c|c|c|c|c|c|c|}
\hline Name of the species & Habit & Life-span & $\begin{array}{l}\text { Fl. and Fr. } \\
\text { time }\end{array}$ & $\begin{array}{l}\text { Raunkiaer's } \\
\text { life-form }\end{array}$ & Sub-type & $\begin{array}{c}\text { Leaf } \\
\text { spectra }\end{array}$ & $\begin{array}{c}\text { IUCN red list } \\
\text { status }\end{array}$ \\
\hline \multicolumn{8}{|l|}{ Piperales Bercht. and J. Presl } \\
\hline \multicolumn{8}{|l|}{ Aristolochiaceae Juss. } \\
\hline Aristolochia indica $\mathrm{L}$. & $\mathrm{C}$ & $\mathrm{A}$ & Jul.-Jan. & $\mathrm{Cr}$ & - & No & $\mathrm{NE}$ \\
\hline \multicolumn{8}{|c|}{ Magnoliales Juss. ex Bercht. and J. Presl } \\
\hline \multicolumn{8}{|c|}{ Annonaceae Juss. } \\
\hline Annona reticulata $\mathrm{L}$. & $\mathrm{T}$ & $\mathrm{P}$ & JuL.-Dec. & $\mathrm{Ph}$ & $\mathrm{N}$ & $\mathrm{Me}$ & $\mathrm{NE}$ \\
\hline Annona squamosa L. & $\mathrm{T}$ & $\mathrm{P}$ & Mar.-Sep. & $\mathrm{Ph}$ & $\mathrm{N}$ & $\mathrm{Me}$ & $\mathrm{LC}$ \\
\hline \multicolumn{8}{|c|}{ Laurales Juss. ex Bercht. and J. Presl } \\
\hline \multicolumn{8}{|l|}{ Lauraceae Juss. } \\
\hline Litsea glutinosa (Lour.) C. B. Rob. & $\mathrm{T}$ & $\mathrm{P}$ & Apr.-Sep. & $\mathrm{Ph}$ & $\mathrm{M}$ & $\mathrm{Ma}$ & LC \\
\hline
\end{tabular}

\section{INDEPENDENT LINEAGE: UNPLACED TO}

MORE INCLUSIVE CLADE

Alismatales R. Br. ex Bercht. and J. Presl

Araceae Juss.

Alocasia macrorrhizos (L.) G. Don

Amorphophallus paeoniifolius (Dennst.) Nicolson

Colocasia esculenta (L.) Schott

Scindapsus officinalis (Roxb.) Schott

Hydrocharitaceae Juss.

Hydrilla verticillata (L. f.) Royle

Dioscoreales Mart.

Dioscoreaceae R. Br.

Dioscorea alata L.

Dioscorea bulbifera L.

Dioscorea pentaphylla L.

Pandanales R. Br. ex Bercht. and J. Presl

Pandanaceae R. Br.

Pandanus odorifer (Forssk.) Kuntze

Liliales Perleb

Colchicaceae DC.

Gloriosa superba L.

Smilacaceae Vent.

Smilax ovalifolia Roxb. ex D. Don

Asparagales Link

Orchidaceae Juss.

Vanda tessellata (Roxb.) Hook. ex G. Don

$\mathrm{H}$
$\mathrm{H}$
$\mathrm{H}$
$\mathrm{C}$

$\begin{array}{cc}\text { P } & \text { Apr.-May } \\ \text { A } & \text { Jun.-Dec. } \\ \text { A } & \text { Jul.-Oct. }\end{array}$

$\begin{array}{lll}\mathrm{Cr} & - & \\ \mathrm{Cr} & - & \\ \mathrm{Cr} & - & \\ \mathrm{Cr} & - & \end{array}$

$\begin{array}{ll}\mathrm{Me} & \mathrm{NE} \\ \mathrm{Mg} & \mathrm{LC} \\ \mathrm{Mg} & \mathrm{LC} \\ \mathrm{Mg} & \mathrm{NE}\end{array}$

$\mathrm{H}$

A Nov.-Mar.

$\mathrm{Cr}$

Le

LC

$\mathrm{C}$

$\mathrm{C}$

P Aug.-Dec.

$\mathrm{Cr} \quad-$

\begin{tabular}{ll}
$\mathrm{Ma}$ & $\mathrm{NE}$ \\
$\mathrm{Ma}$ & $\mathrm{NE}$ \\
$\mathrm{Me}$ & $\mathrm{NE}$ \\
\hline
\end{tabular}

Aug.-Dec.

Sep.-Feb.

$\begin{array}{lll}\mathrm{Cr} & - & \mathrm{M} \\ \mathrm{Cr} & - & \mathrm{M}\end{array}$




\section{Hypoxidaceae R. Br.}

\begin{tabular}{|c|c|c|c|c|c|c|c|}
\hline Name of the species & Habit & Life-span & $\begin{array}{l}\text { Fl. and Fr. } \\
\text { time }\end{array}$ & $\begin{array}{l}\text { Raunkiaer's } \\
\text { life-form }\end{array}$ & Sub-type & $\begin{array}{c}\text { Leaf } \\
\text { spectra }\end{array}$ & $\begin{array}{c}\text { IUCN red list } \\
\text { status }\end{array}$ \\
\hline Curculigo orchioides Gaertn. & $\mathrm{H}$ & $\mathrm{P}$ & Aug.-Oct. & $\mathrm{Cr}$ & - & $\mathrm{Mi}$ & $\mathrm{NE}$ \\
\hline \multicolumn{8}{|l|}{ Xanthorrhoeaceae } \\
\hline Aloe vera (L.) Burm.f. & $\mathrm{H}$ & $\mathrm{P}$ & Dec.-Feb. & $\mathrm{Cr}$ & - & $\mathrm{Me}$ & $\mathrm{NE}$ \\
\hline \multicolumn{8}{|l|}{ Amaryllidaceae J. St.-Hil. } \\
\hline Crinum asiaticum $\mathrm{L}$. & $\mathrm{H}$ & $\mathrm{P}$ & Aug.-Oct. & $\mathrm{Cr}$ & - & $\mathrm{Mg}$ & $\mathrm{NE}$ \\
\hline \multicolumn{8}{|l|}{ Asparagaceae Juss. } \\
\hline Agave americana $\mathrm{L}$. & $\mathrm{S}$ & $\mathrm{P}$ & Sep.-Mar. & $\mathrm{Cr}$ & - & $\mathrm{Mg}$ & $\mathrm{NE}$ \\
\hline Asparagus racemosus Willd. & $\mathrm{C}$ & $\mathrm{P}$ & Aug.-Dec. & $\mathrm{Cr}$ & - & Le & $\mathrm{NE}$ \\
\hline Yucca gloriosa L. & $\mathrm{S}$ & $\mathrm{P}$ & Nov.-Jun. & $\mathrm{Cr}$ & - & $\mathrm{Me}$ & $\mathrm{NE}$ \\
\hline \multicolumn{8}{|l|}{ Arecales Bromhead } \\
\hline \multicolumn{8}{|l|}{ Arecaceae Bercht. and J. Presl } \\
\hline Borassus flabellifer L. & $\mathrm{T}$ & $\mathrm{P}$ & Mar.-Oct. & $\mathrm{Ph}$ & MM & $\mathrm{Mg}$ & EN \\
\hline Phoenix acaulis Roxb. & $\mathrm{S}$ & $\mathrm{P}$ & Feb.-Jun. & $\mathrm{Ch}$ & - & $\mathrm{Me}$ & $\mathrm{NE}$ \\
\hline Phoenix sylvestris (L.) Roxb. & $\mathrm{T}$ & $\mathrm{P}$ & Feb.-Jun. & $\mathrm{Ph}$ & M & $\mathrm{Me}$ & $\mathrm{NE}$ \\
\hline \multicolumn{8}{|l|}{ Commelinales Mirb. ex Bercht. and J. Presl } \\
\hline \multicolumn{8}{|l|}{ Commelinaceae Mirb. } \\
\hline Commelina benghalensis L. & $\mathrm{H}$ & $\mathrm{A}$ & Aug.-Nov. & $\mathrm{Th}$ & - & $\mathrm{Mi}$ & $\mathrm{LC}$ \\
\hline Murdannia nudiflora (L.) Brenan & $\mathrm{H}$ & A & Jul.-Nov. & $\mathrm{Th}$ & - & $\mathrm{Na}$ & $\mathrm{NE}$ \\
\hline \multicolumn{8}{|l|}{ Zingiberales Griseb. } \\
\hline \multicolumn{8}{|l|}{ Costaceae Nakai } \\
\hline Cheilocostus speciosus (J. Koenig) C. D. Specht & $\mathrm{H}$ & $\mathrm{P}$ & Jul.-Sep. & $\mathrm{Cr}$ & - & $\mathrm{Ma}$ & $\mathrm{NE}$ \\
\hline \multicolumn{8}{|l|}{ Zingiberaceae Martinov } \\
\hline Curcuma aromatica Salisb. & $\mathrm{H}$ & $\mathrm{P}$ & May-Jun. & $\mathrm{Cr}$ & - & $\mathrm{Ma}$ & $\mathrm{NE}$ \\
\hline Kaempferia galanga L. & $\mathrm{H}$ & $\mathrm{P}$ & May-Jun. & $\mathrm{Cr}$ & - & $\mathrm{Ma}$ & $\mathrm{NE}$ \\
\hline \multicolumn{8}{|l|}{ Poales Small } \\
\hline \multicolumn{8}{|l|}{ Cyperaceae Juss. } \\
\hline Cyperus difformis $\mathrm{L}$. & $\mathrm{H}$ & $\mathrm{P}$ & Jul.-Nov. & $\mathrm{He}$ & - & Le & $\mathrm{LC}$ \\
\hline Cyperus dubius Rottb. & $\mathrm{H}$ & $\mathrm{p}$ & Sep.-Dec. & $\mathrm{He}$ & - & Le & $\mathrm{LC}$ \\
\hline Cyperus platystylis $\mathrm{R} . \mathrm{Br}$. & $\mathrm{H}$ & $\mathrm{P}$ & May-Jun. & $\mathrm{He}$ & - & Le & $\mathrm{NE}$ \\
\hline Cyperus rotundus L. & $\mathrm{H}$ & $\mathrm{P}$ & Sep.-Dec. & $\mathrm{He}$ & - & Le & $\mathrm{LC}$ \\
\hline Fimbristylis cymosa R. Br. & $\mathrm{H}$ & $\mathrm{p}$ & Feb.-May & $\mathrm{He}$ & - & Le & $\mathrm{LC}$ \\
\hline Fimbristylis dichotoma (L.) Vahl & $\mathrm{H}$ & $\mathrm{P}$ & Aug.-Oct. & $\mathrm{He}$ & - & Le & $\mathrm{LC}$ \\
\hline Fimbristylis quinquangularis (Vahl) Kunth & $\mathrm{H}$ & $\mathrm{P}$ & Aug.-Nov. & $\mathrm{He}$ & - & Le & $\mathrm{LC}$ \\
\hline Rhynchospora colorata (L.) H. Pfeiff. & $\mathrm{H}$ & $\mathrm{P}$ & May-Oct. & $\mathrm{He}$ & - & Le & $\mathrm{NE}$ \\
\hline \multicolumn{8}{|l|}{ Poaceae Barnhart } \\
\hline Aristida setacea Retz. & $\mathrm{H}$ & $\mathrm{P}$ & Aug.-Dec. & $\mathrm{He}$ & - & Le & $\mathrm{NE}$ \\
\hline
\end{tabular}




\begin{tabular}{|c|c|c|c|c|c|c|c|}
\hline Bambusa bambos (L.) Voss & $\mathrm{T}$ & $\mathrm{P}$ & Jul.-Feb. & $\mathrm{Ph}$ & $\mathrm{M}$ & $\mathrm{Me}$ & $\mathrm{LC}$ \\
\hline Brachiaria reptans (L.) C. A. Gardner and C. E. & $\mathrm{H}$ & A & Aug.-Oct. & $\mathrm{He}$ & - & Mi & $\mathrm{LC}$ \\
\hline Name of the species & Habit & Life-span & $\begin{array}{l}\text { Fl. and Fr. } \\
\text { time }\end{array}$ & $\begin{array}{c}\text { Raunkiaer's } \\
\text { life-form }\end{array}$ & Sub-type & $\begin{array}{c}\text { Leaf } \\
\text { spectra }\end{array}$ & $\begin{array}{c}\text { IUCN red list } \\
\text { status }\end{array}$ \\
\hline Chloris barbata (L.) Sw. & $\mathrm{H}$ & $\mathrm{P}$ & Aug.-Nov. & $\mathrm{He}$ & - & Le & $\mathrm{NE}$ \\
\hline Chrysopogon aciculatus (Retz.) Trin. & $\mathrm{H}$ & $\mathrm{P}$ & Sep.-Dec. & $\mathrm{He}$ & - & Le & $\mathrm{NE}$ \\
\hline Chrysopogon zizanioides (L.) Roberty & $\mathrm{H}$ & $\mathrm{P}$ & Jun.-Oct. & $\mathrm{He}$ & - & Le & $\mathrm{NE}$ \\
\hline Coix lacryma-jobi L. & $\mathrm{H}$ & $\mathrm{A}$ & Aug.-Jan. & $\mathrm{He}$ & - & No & $\mathrm{NE}$ \\
\hline Cymbopogon citratus (DC.) Stapf. & $\mathrm{H}$ & $\mathrm{A}$ & Oct.-Dec. & $\mathrm{He}$ & - & Le & $\mathrm{NE}$ \\
\hline Cynodon dactylon (L.) Pers. & $\mathrm{H}$ & $\mathrm{P}$ & All & $\mathrm{He}$ & - & Le & $\mathrm{NE}$ \\
\hline Digitaria sanguinalis (L.) Scop. & $\mathrm{H}$ & $\mathrm{P}$ & Mar.-Jun. & $\mathrm{He}$ & - & Le & $\mathrm{NE}$ \\
\hline Echinochloa crusgalli (L.) P. Beauv. & $\mathrm{H}$ & $\mathrm{A}$ & Aug.-Nov. & $\mathrm{He}$ & - & $\mathrm{Na}$ & $\mathrm{LC}$ \\
\hline Echinochloa frumentacea Link & $\mathrm{H}$ & $\mathrm{A}$ & Aug.-Nov. & $\mathrm{He}$ & - & $\mathrm{Na}$ & $\mathrm{LC}$ \\
\hline Eleusine indica (L.) Gaertn. & $\mathrm{H}$ & $\mathrm{P}$ & Aug.-Nov. & $\mathrm{He}$ & - & Le & $\mathrm{LC}$ \\
\hline Eragrostis amabilis (L.) Wight and Am. & $\mathrm{H}$ & $\mathrm{P}$ & Aug.-Feb. & $\mathrm{He}$ & - & Le & $\mathrm{NE}$ \\
\hline Eragrostis ciliaris (L.) R. Br. & $\mathrm{H}$ & $\mathrm{P}$ & Aug.-Feb. & $\mathrm{He}$ & - & Le & $\mathrm{NE}$ \\
\hline Imperata cylindrica (L.) Raeusch. & $\mathrm{H}$ & $\mathrm{P}$ & Oct.-Dec. & $\mathrm{He}$ & - & $\mathrm{Na}$ & $\mathrm{LC}$ \\
\hline Paspalum scrobiculatum L. & $\mathrm{H}$ & $\mathrm{P}$ & Aug.-Nov. & $\mathrm{He}$ & - & $\mathrm{Na}$ & $\mathrm{LC}$ \\
\hline Pennisetum glaucum (L.) R. Br. & $\mathrm{H}$ & $\mathrm{P}$ & Aug.-Oct. & $\mathrm{He}$ & - & Mi & $\mathrm{LC}$ \\
\hline Setaria glauca (L.) R. Br. & $\mathrm{H}$ & $\mathrm{P}$ & Aug.-Nov. & $\mathrm{He}$ & - & Le & $\mathrm{NE}$ \\
\hline Sporobolus indicus (L.) R. Br. & $\mathrm{H}$ & $\mathrm{P}$ & Aug.-Nov. & $\mathrm{He}$ & - & $\mathrm{Na}$ & $\mathrm{NE}$ \\
\hline \multicolumn{8}{|l|}{ EUDICOTS } \\
\hline \multicolumn{8}{|l|}{ Ranunculales Juss. ex Bercht. and J. Presl } \\
\hline \multicolumn{8}{|l|}{ Papaveraceae Juss. } \\
\hline Argemone mexicana $\mathrm{L}$. & $\mathrm{H}$ & A & Dec.-Apr. & Th & - & $\mathrm{Ma}$ & $\mathrm{NE}$ \\
\hline \multicolumn{8}{|l|}{ Menispermaceae Juss. } \\
\hline Stephania japonica (Thunb.) Mier. & $\mathrm{C}$ & $\mathrm{P}$ & Jul.-Dec. & $\mathrm{Ph}$ & $\mathrm{N}$ & $\mathrm{Me}$ & $\mathrm{NE}$ \\
\hline Tinospora sinensis (Lour.) Merr. & $\mathrm{C}$ & $\mathrm{P}$ & Feb.-Jun. & $\mathrm{Ph}$ & $\mathrm{N}$ & $\mathrm{Me}$ & $\mathrm{NE}$ \\
\hline \multicolumn{8}{|l|}{ CORE EUDICOTS } \\
\hline \multicolumn{8}{|l|}{ SUPERROSIDS } \\
\hline \multicolumn{8}{|l|}{ Saxifragales Bercht. and J. Presl } \\
\hline \multicolumn{8}{|l|}{ Crassulaceae J. St. -Hil. } \\
\hline Bryophyllum pinnatum (Lam.) Oken & $\mathrm{H}$ & $\mathrm{P}$ & Mar.-Jun. & $\mathrm{Ch}$ & - & $\mathrm{Ma}$ & $\mathrm{NE}$ \\
\hline \multicolumn{8}{|l|}{ ROSIDS } \\
\hline \multicolumn{8}{|l|}{ Vitales Juss. ex Bercht. and J. Presl } \\
\hline \multicolumn{8}{|l|}{ Vitaceae Juss. } \\
\hline Ampelocissus latifolia (Roxb.) Planch. & $\mathrm{C}$ & $\mathrm{P}$ & Jun.- Sep. & $\mathrm{Ph}$ & $\mathrm{N}$ & $\mathrm{Me}$ & $\mathrm{NE}$ \\
\hline Cayratia trifolia (L.) Domin. & $\mathrm{C}$ & $\mathrm{P}$ & Aug.-Dec. & $\mathrm{Ph}$ & $\mathrm{N}$ & No & NE \\
\hline Cissus quadrangularis $\mathrm{L}$. & $\mathrm{C}$ & $\mathrm{P}$ & Jul.-Jan. & $\mathrm{Ph}$ & $\mathrm{N}$ & No & $\mathrm{NE}$ \\
\hline
\end{tabular}


Sen and Bhakat/ Journal of Tropical Forestry and Environment Vol. 10 No. 01 (2020) 17-39

\begin{tabular}{|c|c|c|c|c|c|c|c|}
\hline Leea asiatica (L.) Ridsdale & $\mathrm{C}$ & $\mathrm{P}$ & Jul.-Sep. & $\mathrm{Ph}$ & $\mathrm{N}$ & $\mathrm{Me}$ & $\mathrm{NE}$ \\
\hline \multicolumn{8}{|l|}{ Zygophyllales Link } \\
\hline \multicolumn{8}{|l|}{ Zygophyllaceae R. Br. } \\
\hline Name of the species & Habit & Life-span & $\begin{array}{c}\text { Fl. and Fr. } \\
\text { time }\end{array}$ & $\begin{array}{c}\text { Raunkiaer's } \\
\text { life-form }\end{array}$ & Sub-type & $\begin{array}{c}\text { Leaf } \\
\text { spectra }\end{array}$ & $\begin{array}{c}\text { IUCN red list } \\
\text { status }\end{array}$ \\
\hline Tribulus terrestris L. & $\mathrm{C}$ & $\mathrm{A}$ & Feb.-Sep. & Th & - & $\mathrm{Na}$ & $\mathrm{LC}$ \\
\hline \multicolumn{8}{|l|}{ Fabales Bromhead } \\
\hline \multicolumn{8}{|l|}{ Fabaceae Lindl. } \\
\hline Abrus precatorius L. & $\mathrm{C}$ & $\mathrm{P}$ & Aug.-Mar. & $\mathrm{Ph}$ & $\mathrm{N}$ & $\mathrm{Na}$ & $\mathrm{NE}$ \\
\hline Acacia pennata (L.) Willd. & $\mathrm{T}$ & $\mathrm{P}$ & Feb.-Oct & $\mathrm{Ph}$ & M & $\mathrm{Na}$ & $\mathrm{LC}$ \\
\hline Acacia polyacantha Willd. & $\mathrm{T}$ & $\mathrm{P}$ & Feb.-Oct & $\mathrm{Ph}$ & $\mathrm{M}$ & $\mathrm{Na}$ & $\mathrm{LC}$ \\
\hline Acacia rugata (Lam.) Fawc. and Rendle & $\mathrm{T}$ & $\mathrm{P}$ & Feb.-Oct & $\mathrm{Ph}$ & $\mathrm{M}$ & $\mathrm{Na}$ & $\mathrm{NE}$ \\
\hline Acacia auriculiformis Benth. & $\mathrm{T}$ & $\mathrm{P}$ & Feb.-Oct. & $\mathrm{Ph}$ & $\mathrm{M}$ & $\mathrm{Me}$ & $\mathrm{LC}$ \\
\hline Acacia nilotica (L.) Delile & $\mathrm{T}$ & $\mathrm{P}$ & Jun.-Sep. & $\mathrm{Ph}$ & $\mathrm{M}$ & $\mathrm{Na}$ & $\mathrm{LC}$ \\
\hline Adenanthera pavonina $\mathrm{L}$. & $\mathrm{T}$ & $\mathrm{P}$ & Mar.-Jan. & $\mathrm{Ph}$ & $\mathrm{M}$ & No & $\mathrm{LC}$ \\
\hline Albizia lebbeck (L.) Benth. & $\mathrm{T}$ & $\mathrm{P}$ & Mar.-Feb. & $\mathrm{Ph}$ & $\mathrm{MM}$ & $\mathrm{Mi}$ & $\mathrm{NE}$ \\
\hline Albizia saman (Jacq.) Merr. & $\mathrm{T}$ & $\mathrm{P}$ & Mar.-Feb. & $\mathrm{Ph}$ & $\mathrm{MM}$ & $\mathrm{Me}$ & $\mathrm{NE}$ \\
\hline Bauhinia vahlii Wight and Arn. & $\mathrm{C}$ & $\mathrm{P}$ & Apr.-Feb. & $\mathrm{Ph}$ & $\mathrm{N}$ & $\mathrm{Mg}$ & $\mathrm{NE}$ \\
\hline Bauhinia variegate $\mathrm{L}$. & $\mathrm{T}$ & $\mathrm{P}$ & Feb.-Jun. & $\mathrm{Ph}$ & $\mathrm{M}$ & $\mathrm{Me}$ & $\mathrm{LC}$ \\
\hline Butea superba Roxb. & $\mathrm{C}$ & $\mathrm{P}$ & Feb.-Jul. & $\mathrm{Ph}$ & M & $\mathrm{Ma}$ & $\mathrm{NE}$ \\
\hline Caesalpinia pulcherrima (L.) Sw. & $\mathrm{T}$ & $\mathrm{P}$ & Mar.-Sep. & $\mathrm{Ph}$ & M & $\mathrm{Mi}$ & $\mathrm{LC}$ \\
\hline Caesalpinia bonduc (L.) Roxb. & $\mathrm{C}$ & $\mathrm{P}$ & Aug.-Apr. & $\mathrm{Ph}$ & $\mathrm{N}$ & $\mathrm{Mi}$ & $\mathrm{LC}$ \\
\hline Caesalpinia globulorum Bakh. f. and P. Royen & $\mathrm{C}$ & $\mathrm{P}$ & Mar.-Sep. & $\mathrm{Ph}$ & $\mathrm{N}$ & $\mathrm{Mi}$ & $\mathrm{NE}$ \\
\hline Cajanus scarabaeoides (L.) Thouars & $\mathrm{C}$ & A & Sep.-Feb. & $\mathrm{Ph}$ & $\mathrm{N}$ & $\mathrm{Mi}$ & $\mathrm{LC}$ \\
\hline Clitoria ternatea $\mathrm{L}$. & $\mathrm{C}$ & A & All & $\mathrm{Ph}$ & $\mathrm{N}$ & No & $\mathrm{NE}$ \\
\hline Codariocalyx motorius (Houtt.) H. Ohashi & $\mathrm{S}$ & $\mathrm{A}$ & Aug.-Dec. & $\mathrm{Ch}$ & - & $\mathrm{Na}$ & $\mathrm{NE}$ \\
\hline Crotalaria pallida Aiton & $\mathrm{S}$ & A & Aug.-Jan. & $\mathrm{Ch}$ & - & No & $\mathrm{NE}$ \\
\hline Crotalaria prostrata Willd. & $\mathrm{H}$ & A & Aug.-Jan. & Th & - & No & $\mathrm{NE}$ \\
\hline Derris indica (Lam.) Bennet & $\mathrm{C}$ & $\mathrm{P}$ & Jul.-Jan. & $\mathrm{Ph}$ & $\mathrm{N}$ & $\mathrm{Na}$ & $\mathrm{NE}$ \\
\hline Flemingia strobilifera (L.) W. T. Aiton & $\mathrm{H}$ & $\mathrm{A}$ & Feb.-Sep. & $\mathrm{Ch}$ & - & $\mathrm{Me}$ & $\mathrm{NE}$ \\
\hline Indigofera tinctoria $\mathrm{L}$. & $\mathrm{H}$ & $\mathrm{B}$ & Aug.-Nov. & Th & - & $\mathrm{Mi}$ & $\mathrm{NE}$ \\
\hline Mimosa pudica L. & $\mathrm{H}$ & $\mathrm{P}$ & Jul.-Nov. & Th & - & $\mathrm{Na}$ & $\mathrm{LC}$ \\
\hline Mimosa rubicaulis Lam. & $\mathrm{S}$ & $\mathrm{P}$ & Jul.-Nov. & $\mathrm{Ch}$ & - & $\mathrm{Na}$ & $\mathrm{NE}$ \\
\hline Mucuna pruriens (L.) DC. & $\mathrm{C}$ & $\mathrm{A}$ & Sep.-May & $\mathrm{Ch}$ & - & Le & $\mathrm{NE}$ \\
\hline Parkinsonia aculeate L. & $\mathrm{T}$ & $\mathrm{P}$ & Oct.-Jun. & $\mathrm{Ph}$ & $\mathrm{N}$ & $\mathrm{Mi}$ & $\mathrm{LC}$ \\
\hline Peltophorum pterocarpum (DC.) K. Heyne & $\mathrm{T}$ & $\mathrm{P}$ & Mar.-Jan. & $\mathrm{Ph}$ & MM & $\mathrm{Mi}$ & $\mathrm{NE}$ \\
\hline Pongamia pinnata (L.) Pierre & $\mathrm{T}$ & $\mathrm{P}$ & Apr.-Feb. & $\mathrm{Ph}$ & $\mathrm{M}$ & $\mathrm{Me}$ & $\mathrm{LC}$ \\
\hline Senna alata (L.) Roxb. & $\mathrm{S}$ & $\mathrm{A}$ & Aug.-Nov. & $\mathrm{Ch}$ & - & $\mathrm{Ma}$ & $\mathrm{LC}$ \\
\hline Senna occidentalis (L.) Link & $\mathrm{S}$ & $\mathrm{P}$ & Aug.-Dec. & $\mathrm{Ch}$ & - & No & $\mathrm{NE}$ \\
\hline
\end{tabular}




\begin{tabular}{|c|c|c|c|c|c|c|c|}
\hline Senna tora (L.) Roxb. & $\mathrm{H}$ & $\mathrm{A}$ & Sep.-Dec. & Th & - & $\mathrm{Mi}$ & NE \\
\hline Sesbania sesban (L.) Merr. & $\mathrm{S}$ & $\mathrm{P}$ & Dec.-Apr. & $\mathrm{Ch}$ & - & $\mathrm{Ma}$ & $\mathrm{LC}$ \\
\hline Tamarindus indica $\mathrm{L}$. & $\mathrm{T}$ & $\mathrm{P}$ & Apr.-Jan. & $\mathrm{Ph}$ & MM & $\mathrm{Na}$ & $\mathrm{LC}$ \\
\hline Tephrosia purpurea (L.) Pers. & $\mathrm{H}$ & $\mathrm{P}$ & Sep.-Dec. & $\mathrm{Th}$ & - & $\mathrm{Na}$ & $\mathrm{LC}$ \\
\hline Name of the species & Habit & Life-span & $\begin{array}{l}\text { Fl. and Fr. } \\
\text { time }\end{array}$ & $\begin{array}{l}\text { Raunkiaer's } \\
\text { life-form }\end{array}$ & Sub-type & $\begin{array}{c}\text { Leaf } \\
\text { spectra }\end{array}$ & $\begin{array}{c}\text { IUCN red list } \\
\text { status }\end{array}$ \\
\hline Zornia gibbosa Span. & $\mathrm{H}$ & A & Aug.-Nov. & Th & - & $\mathrm{Na}$ & $\mathrm{NE}$ \\
\hline \multicolumn{8}{|l|}{ Polygalaceae Hoffmanns. and Link } \\
\hline Polygala arvensis Willd. & $\mathrm{H}$ & A & Jul.-Dec. & $\mathrm{Th}$ & - & $\mathrm{Me}$ & $\mathrm{NE}$ \\
\hline \multicolumn{8}{|l|}{ Rosales Bercht. and J.Presl } \\
\hline \multicolumn{8}{|l|}{ Rhamnaceae Juss. } \\
\hline Ventilago denticulata Willd. & $\mathrm{C}$ & $\mathrm{P}$ & Sep.-Jun. & $\mathrm{Ph}$ & $\mathrm{N}$ & $\mathrm{Me}$ & $\mathrm{NE}$ \\
\hline Ziziphus jujube Mill. & $\mathrm{T}$ & $\mathrm{P}$ & Sep.-Mar. & $\mathrm{Ph}$ & $\mathrm{M}$ & No & $\mathrm{LC}$ \\
\hline Ziziphus oenopolia (L.) Mill. & $\mathrm{C}$ & $\mathrm{P}$ & Nov.-Mar. & $\mathrm{Ph}$ & $\mathrm{N}$ & No & $\mathrm{LC}$ \\
\hline \multicolumn{8}{|l|}{ Ulmaceae Mirb. } \\
\hline Holoptelea integrifolia Planch & $\mathrm{T}$ & $\mathrm{P}$ & Jan.-Jun. & $\mathrm{Ph}$ & MM & $\mathrm{Me}$ & $\mathrm{NE}$ \\
\hline \multicolumn{8}{|l|}{ Moraceae Gaudich. } \\
\hline Artocarpus heterophyllus Lamk. & $\mathrm{T}$ & $\mathrm{P}$ & Jan.-Aug. & $\mathrm{Ph}$ & $\mathrm{M}$ & $\mathrm{Ma}$ & $\mathrm{NE}$ \\
\hline Ficus benghalensis L. & $\mathrm{T}$ & $\mathrm{P}$ & Mar.-Sep. & $\mathrm{Ph}$ & MM & $\mathrm{Ma}$ & $\mathrm{NE}$ \\
\hline Ficus religiosa $\mathrm{L}$. & $\mathrm{T}$ & $\mathrm{P}$ & Jun.-Aug. & $\mathrm{Ph}$ & MM & $\mathrm{Ma}$ & $\mathrm{NE}$ \\
\hline Ficus racemose $\mathrm{L}$. & $\mathrm{T}$ & $\mathrm{P}$ & Mar.-Aug. & $\mathrm{Ph}$ & $\mathrm{M}$ & $\mathrm{Ma}$ & $\mathrm{LC}$ \\
\hline Streblus asper Lour. & $\mathrm{T}$ & $\mathrm{P}$ & Feb.-Jun. & $\mathrm{Ph}$ & $\mathrm{N}$ & $\mathrm{Mi}$ & $\mathrm{LC}$ \\
\hline \multicolumn{8}{|l|}{ Cucurbitales Juss. ex Bercht. and J. Presl } \\
\hline \multicolumn{8}{|l|}{ Cucurbitaceae Juss. } \\
\hline Cayaponia laciniosa (L.) C. Jeffrey & $\mathrm{C}$ & A & Jun.-Jan. & $\mathrm{Ph}$ & $\mathrm{N}$ & $\mathrm{Mi}$ & NE \\
\hline Coccinia grandis (L.) Voigt & $\mathrm{C}$ & $\mathrm{P}$ & Mar.-Dec. & $\mathrm{Ph}$ & $\mathrm{N}$ & $\mathrm{Me}$ & $\mathrm{NE}$ \\
\hline Momordica dioica Roxb. ex Willd. & $\mathrm{C}$ & A & Aug.-Dec. & $\mathrm{Ph}$ & $\mathrm{N}$ & $\mathrm{Me}$ & NE \\
\hline Trichosanthes cucumerina $\mathrm{L}$ & $\mathrm{C}$ & $\mathrm{P}$ & Aug.-Dec. & $\mathrm{Ph}$ & $\mathrm{N}$ & $\mathrm{Me}$ & $\mathrm{NE}$ \\
\hline Trichosanthes tricuspidata Lour. & $\mathrm{C}$ & A & Apr.-Sep. & $\mathrm{Ph}$ & $\mathrm{N}$ & $\mathrm{Me}$ & $\mathrm{NE}$ \\
\hline \multicolumn{8}{|l|}{ Celastrales Link } \\
\hline \multicolumn{8}{|l|}{ Celastraceae R. Br. } \\
\hline Celastrus paniculatus Willd. & $\mathrm{C}$ & $\mathrm{P}$ & Apr.-Dec. & $\mathrm{Ph}$ & $\mathrm{N}$ & $\mathrm{Me}$ & $\mathrm{NE}$ \\
\hline \multicolumn{8}{|l|}{ Oxalidales Bercht. and J. Presl } \\
\hline \multicolumn{8}{|l|}{ Oxalidaceae R.Br. } \\
\hline Averrhoa carambola L. & $\mathrm{T}$ & $\mathrm{P}$ & Feb.-Sep. & $\mathrm{Ph}$ & $\mathrm{N}$ & $\mathrm{Ma}$ & $\mathrm{NE}$ \\
\hline Oxalis corniculata $\mathrm{L}$. & $\mathrm{H}$ & A & All & Th & - & $\mathrm{Na}$ & $\mathrm{NE}$ \\
\hline \multicolumn{8}{|l|}{ Malpighiales Juss. ex Bercht. and J. Presl } \\
\hline \multicolumn{8}{|l|}{ Clusiaceae Lindl. } \\
\hline Garcinia xanthochymus Hook. f. ex T. Anderson & $\mathrm{T}$ & $\mathrm{P}$ & Mar.-Jan. & $\mathrm{Ph}$ & $\mathrm{M}$ & No & $\mathrm{NE}$ \\
\hline
\end{tabular}




\begin{tabular}{|c|c|c|c|c|c|c|c|}
\hline Violaceae Batsch & & & & & & & \\
\hline Hybanthus enneaspermus (L.) F. Muell. & $\mathrm{H}$ & $\mathrm{P}$ & Jul.-Nov. & Th & - & $\mathrm{Na}$ & NE \\
\hline \multicolumn{8}{|l|}{ Salicaceae Mirb. } \\
\hline Flacourtia indica (Burm. f.) Merr. & $\mathrm{S}$ & $\mathrm{P}$ & Sep.-May. & $\mathrm{Ch}$ & - & $\mathrm{Mi}$ & LC \\
\hline Name of the species & Habit & Life-span & $\begin{array}{l}\text { Fl. and Fr. } \\
\text { time }\end{array}$ & $\begin{array}{c}\text { Raunkiaer's } \\
\text { life-form }\end{array}$ & Sub-type & $\begin{array}{c}\text { Leaf } \\
\text { spectra }\end{array}$ & $\begin{array}{l}\text { IUCN red list } \\
\text { status }\end{array}$ \\
\hline \multicolumn{8}{|l|}{ Euphorbiaceae Juss. } \\
\hline Acalypha indica $\mathrm{L}$. & $\mathrm{H}$ & $\mathrm{A}$ & All & Th & - & No & $\mathrm{NE}$ \\
\hline Croton bonplandianus Baill. & $\mathrm{H}$ & $\mathrm{P}$ & All & Th & - & No & $\mathrm{NE}$ \\
\hline Euphorbia antiquorum L. & $\mathrm{T}$ & $\mathrm{P}$ & Jan.-Apr. & $\mathrm{Ph}$ & $\mathrm{N}$ & Le & $\mathrm{NE}$ \\
\hline Euphorbia hirta L. & $\mathrm{H}$ & A & Feb.-Dec. & $\mathrm{Th}$ & - & $\mathrm{Na}$ & $\mathrm{NE}$ \\
\hline Euphorbia neriifolia L. & $\mathrm{S}$ & $\mathrm{P}$ & Oct.-Feb. & $\mathrm{Ch}$ & - & $\mathrm{Le}$ & $\mathrm{NE}$ \\
\hline Jatropha curcas L. & $\mathrm{S}$ & $\mathrm{P}$ & Mar.-Aug. & $\mathrm{Ch}$ & - & $\mathrm{Ma}$ & $\mathrm{NE}$ \\
\hline Jatropha gossypifolia L. & $\mathrm{S}$ & $\mathrm{P}$ & Mar.-Aug. & $\mathrm{Ch}$ & - & $\mathrm{Ma}$ & NE \\
\hline Ricinus communis L. & $\mathrm{S}$ & $\mathrm{P}$ & Jan.-Apr. & $\mathrm{Ph}$ & $\mathrm{N}$ & $\mathrm{Mg}$ & $\mathrm{NE}$ \\
\hline Tragia involucrata L. & $\mathrm{C}$ & $\mathrm{P}$ & Mar.-Jan. & $\mathrm{Ph}$ & $\mathrm{N}$ & $\mathrm{Me}$ & $\mathrm{NE}$ \\
\hline \multicolumn{8}{|l|}{ Phyllanthaceae Martinov } \\
\hline Antidesma acidum Retz. & $\mathrm{T}$ & $\mathrm{P}$ & Jun.-Dec. & $\mathrm{Ph}$ & $\mathrm{N}$ & $\mathrm{Me}$ & $\mathrm{LC}$ \\
\hline Breynia vitis-idaea (Burm. f.) C. E. C. Fisch. & $\mathrm{S}$ & $\mathrm{P}$ & Apr.-Dec. & $\mathrm{Ph}$ & $\mathrm{N}$ & $\mathrm{Mi}$ & $\mathrm{LC}$ \\
\hline Bridelia retusa (L.) A. Juss. & $\mathrm{T}$ & $\mathrm{P}$ & Mar.-Dec. & $\mathrm{Ph}$ & $\mathrm{N}$ & $\mathrm{Me}$ & $\mathrm{LC}$ \\
\hline Phyllanthus amarus Schumach. and Thonn. & $\mathrm{H}$ & $\mathrm{A}$ & Apr.-Sep. & $\mathrm{Th}$ & - & $\mathrm{Na}$ & $\mathrm{NE}$ \\
\hline Phyllanthus virgatus G. Forst. & $\mathrm{H}$ & $\mathrm{A}$ & Apr.-Sep. & Th & - & $\mathrm{Na}$ & $\mathrm{NE}$ \\
\hline \multicolumn{8}{|l|}{ Myrtales Juss. ex Bercht. and J. Presl } \\
\hline \multicolumn{8}{|l|}{ Combretaceae R. Br. } \\
\hline Combretum album Pers. & $\mathrm{C}$ & $\mathrm{P}$ & Nov.-May & $\mathrm{Ph}$ & $\mathrm{N}$ & $\mathrm{Me}$ & $\mathrm{NE}$ \\
\hline Combretum decandrum Jacq. & $\mathrm{C}$ & $\mathrm{P}$ & Nov.-May & $\mathrm{Ph}$ & $\mathrm{N}$ & $\mathrm{Me}$ & $\mathrm{NE}$ \\
\hline Combretum indicum (L.) De Filipps & $\mathrm{C}$ & $\mathrm{P}$ & All & $\mathrm{Ph}$ & $\mathrm{N}$ & $\mathrm{Me}$ & NE \\
\hline $\begin{array}{l}\text { Terminalia arjuna (Roxb. ex DC.) Wight and } \\
\text { Arn. }\end{array}$ & $\mathrm{T}$ & $\mathrm{P}$ & Apr.-Feb. & $\mathrm{Ph}$ & MM & $\mathrm{Ma}$ & NE \\
\hline Terminalia bellirica (Gaertn.) Roxb. & $\mathrm{T}$ & $\mathrm{P}$ & Apr.-Feb. & $\mathrm{Ph}$ & $\mathrm{MM}$ & $\mathrm{Ma}$ & $\mathrm{NE}$ \\
\hline Terminalia tomentosa (Roxb.) Wight and Arn. & $\mathrm{T}$ & $\mathrm{P}$ & Apr.-May & $\mathrm{Ph}$ & $\mathrm{MM}$ & $\mathrm{Ma}$ & NE \\
\hline \multicolumn{8}{|l|}{ Lythraceae J.St.-Hil. } \\
\hline Ammannia baccifera $\mathrm{L}$. & $\mathrm{H}$ & $\mathrm{A}$ & Sep.-Feb. & $\mathrm{Th}$ & - & $\mathrm{Le}$ & $\mathrm{LC}$ \\
\hline Lawsonia inermis L. & $\mathrm{S}$ & $\mathrm{P}$ & Oct.-Dec. & $\mathrm{Ph}$ & $\mathrm{N}$ & $\mathrm{Na}$ & $\mathrm{NE}$ \\
\hline Punica granatum L. & $\mathrm{S}$ & $\mathrm{P}$ & Mar.-Oct. & $\mathrm{Ph}$ & $\mathrm{N}$ & $\mathrm{Na}$ & $\mathrm{LC}$ \\
\hline Rotala densiflora (Roth) Koehne & $\mathrm{H}$ & A & Aug.-Nov. & Th & - & Le & $\mathrm{LC}$ \\
\hline \multicolumn{8}{|l|}{ Onagraceae Juss. } \\
\hline Ludwigia octovalvis (Jacq.) P. H. Raven & $\mathrm{H}$ & $\mathrm{A}$ & Sep.-Jan. & Th & - & $\mathrm{Mi}$ & LC \\
\hline Myrtaceae Juss. & & & & & & & \\
\hline
\end{tabular}




\begin{tabular}{|c|c|c|c|c|c|c|c|}
\hline Eucalyptus tereticornis $\mathrm{Sm}$. & $\mathrm{T}$ & $\mathrm{P}$ & Mar.-Jul. & $\mathrm{Ph}$ & MM & $\mathrm{Me}$ & NE \\
\hline Psidium guajava L. & $\mathrm{T}$ & $\mathrm{P}$ & Apr.-Oct. & $\mathrm{Ph}$ & $\mathrm{N}$ & $\mathrm{Me}$ & $\mathrm{LC}$ \\
\hline Syzygium cumini (L.) Skeels & $\mathrm{T}$ & $\mathrm{P}$ & Mar.-Jul. & $\mathrm{Ph}$ & $\mathrm{MM}$ & $\mathrm{Me}$ & LC \\
\hline \multicolumn{8}{|l|}{ Melastomataceae Juss. } \\
\hline Melastoma malabathricum L. & $\mathrm{S}$ & $\mathrm{P}$ & May-Jan. & $\mathrm{Ch}$ & - & $\mathrm{Me}$ & $\mathrm{NE}$ \\
\hline Name of the species & Habit & Life-span & $\begin{array}{l}\text { Fl. and Fr. } \\
\text { time }\end{array}$ & $\begin{array}{c}\text { Raunkiaer's } \\
\text { life-form }\end{array}$ & Sub-type & $\begin{array}{c}\text { Leaf } \\
\text { spectra }\end{array}$ & $\begin{array}{c}\text { IUCN red list } \\
\text { status }\end{array}$ \\
\hline \multicolumn{8}{|l|}{ Sapindales Juss. ex Bercht. and J. Presl } \\
\hline \multicolumn{8}{|l|}{ Anacardiaceae R. Br. } \\
\hline Lannea coromandelica (Houtt.) Merr. & $\mathrm{T}$ & $\mathrm{P}$ & Feb.-Jun. & $\mathrm{Ph}$ & $\mathrm{M}$ & $\mathrm{Mg}$ & $\mathrm{NE}$ \\
\hline Mangifera indica L. & $\mathrm{T}$ & $\mathrm{P}$ & Feb.-Jun. & $\mathrm{Ph}$ & $\mathrm{M}$ & $\mathrm{Ma}$ & $\mathrm{DD}$ \\
\hline Semecarpus anacardium L. f. & $\mathrm{T}$ & $\mathrm{P}$ & Jul.-Dec. & $\mathrm{Ph}$ & $\mathrm{M}$ & $\mathrm{Mg}$ & $\mathrm{NE}$ \\
\hline \multicolumn{8}{|l|}{ Sapindaceae Juss. } \\
\hline Cardiospermum halicacabum L. & $\mathrm{C}$ & $\mathrm{A}$ & Jul.-Dec. & $\mathrm{Ph}$ & $\mathrm{N}$ & No & $\mathrm{NE}$ \\
\hline Sapindus emarginatus Vahl & $\mathrm{T}$ & $\mathrm{P}$ & Dec.-May & $\mathrm{Ph}$ & $\mathrm{M}$ & $\mathrm{Me}$ & $\mathrm{NE}$ \\
\hline Schleichera oleosa (Lour.) Merr. & $\mathrm{T}$ & $\mathrm{P}$ & Mar.-Jul. & $\mathrm{Ph}$ & $\mathrm{MM}$ & $\mathrm{Ma}$ & $\mathrm{LC}$ \\
\hline \multicolumn{8}{|l|}{ Rutaceae Juss. } \\
\hline Aegle marmelos (L.) Corrêa & $\mathrm{T}$ & $\mathrm{P}$ & May-Jul. & $\mathrm{Ph}$ & $\mathrm{M}$ & $\mathrm{Me}$ & $\mathrm{NE}$ \\
\hline Glycosmis pentaphylla (Retz.) DC. & $\mathrm{T}$ & $\mathrm{P}$ & Sep.-Feb. & $\mathrm{Ph}$ & $\mathrm{N}$ & $\mathrm{Me}$ & $\mathrm{LC}$ \\
\hline Limonia acidissima Groff & $\mathrm{T}$ & $\mathrm{P}$ & Jan.-Dec. & $\mathrm{Ph}$ & $\mathrm{M}$ & $\mathrm{Na}$ & $\mathrm{NE}$ \\
\hline Murraya koenigii (L.) Spreng. & $\mathrm{T}$ & $\mathrm{P}$ & Apr.-Jun. & $\mathrm{Ph}$ & $\mathrm{N}$ & $\mathrm{Na}$ & $\mathrm{NE}$ \\
\hline \multicolumn{8}{|l|}{ Simaroubaceae DC. } \\
\hline Ailanthus excelsa Roxb. & $\mathrm{T}$ & $\mathrm{P}$ & Jan.-Jun. & $\mathrm{Ph}$ & $\mathrm{MM}$ & $\mathrm{Ma}$ & $\mathrm{NE}$ \\
\hline \multicolumn{8}{|l|}{ Meliaceae Juss. } \\
\hline Azadirachta indica A. Juss. & $\mathrm{T}$ & $\mathrm{P}$ & Mar.-Jul. & $\mathrm{Ph}$ & $\mathrm{M}$ & No & $\mathrm{LC}$ \\
\hline Melia azedarach L. & $\mathrm{T}$ & $\mathrm{P}$ & Feb.-Nov. & $\mathrm{Ph}$ & $\mathrm{M}$ & No & $\mathrm{LC}$ \\
\hline \multicolumn{8}{|l|}{ Malvales Juss. ex Bercht. and J. Presl } \\
\hline \multicolumn{8}{|l|}{ Malvaceae Juss. } \\
\hline Abelmoschus crinitus Wall. & $\mathrm{S}$ & $\mathrm{A}$ & Mar.-Sep. & $\mathrm{Ch}$ & - & No & $\mathrm{NE}$ \\
\hline Abelmoschus moschatus Medik. & $\mathrm{S}$ & $\mathrm{A}$ & Mar.-Aug.. & $\mathrm{Ch}$ & - & No & $\mathrm{NE}$ \\
\hline Abutilon indicum (L.) Sweet & $\mathrm{S}$ & $\mathrm{A}$ & Jun.-Dec. & $\mathrm{Ch}$ & - & $\mathrm{Ma}$ & $\mathrm{NE}$ \\
\hline Ambroma augusta L. f. & $\mathrm{S}$ & $\mathrm{P}$ & Jan.-Mar. & $\mathrm{Ph}$ & $\mathrm{N}$ & $\mathrm{Ma}$ & $\mathrm{NE}$ \\
\hline Azanza lampas (Cav.) Alef. & $\mathrm{S}$ & $\mathrm{A}$ & Sep.-Dec. & $\mathrm{Ch}$ & - & $\mathrm{Ma}$ & $\mathrm{NE}$ \\
\hline Corchorus aestuans L. & $\mathrm{H}$ & $\mathrm{A}$ & Jul.-Nov. & Th & - & $\mathrm{Me}$ & $\mathrm{NE}$ \\
\hline Gossypium arboreum L. & $\mathrm{S}$ & $\mathrm{P}$ & Aug.-Feb. & $\mathrm{Ch}$ & - & $\mathrm{Ma}$ & NT \\
\hline Gossypium barbadense L. & $\mathrm{S}$ & $\mathrm{P}$ & Dec.-Apr. & $\mathrm{Ch}$ & - & $\mathrm{Ma}$ & $\mathrm{LC}$ \\
\hline Grewia helicterifolia Wall, ex G. Don & $\mathrm{T}$ & $\mathrm{P}$ & Jun.-Sep. & $\mathrm{Ph}$ & $\mathrm{N}$ & $\mathrm{Ma}$ & $\mathrm{NE}$ \\
\hline Grewia asiatica L. & $\mathrm{T}$ & $\mathrm{P}$ & Jun.-Aug. & $\mathrm{Ph}$ & $\mathrm{N}$ & $\mathrm{Me}$ & $\mathrm{LC}$ \\
\hline Helicteres isora $\mathrm{L}$. & $\mathrm{S}$ & $\mathrm{P}$ & Sep.-Feb. & $\mathrm{Ph}$ & $\mathrm{N}$ & $\mathrm{Me}$ & $\mathrm{NE}$ \\
\hline Hibiscus rosa-sinensis L. & $\mathrm{S}$ & $\mathrm{P}$ & All & $\mathrm{Ch}$ & - & $\mathrm{Me}$ & NE \\
\hline
\end{tabular}


Sen and Bhakat/ Journal of Tropical Forestry and Environment Vol. 10 No. 01 (2020) 17-39

\begin{tabular}{|c|c|c|c|c|c|c|c|}
\hline Hibiscus vitifolius L. & $\mathrm{S}$ & A & Oct.-Feb. & $\mathrm{Ch}$ & - & $\mathrm{Me}$ & $\mathrm{NE}$ \\
\hline Pterospermum acerifolium (L.) Willd. & $\mathrm{T}$ & $\mathrm{P}$ & Jan.-Aug. & $\mathrm{Ph}$ & MM & $\mathrm{Ma}$ & $\mathrm{NE}$ \\
\hline Sida acuta Burm. f. & $\mathrm{S}$ & A & Aug.-Dec. & Th & - & No & $\mathrm{NE}$ \\
\hline Sida cordata (Burm.f.) Borss. Waalk. & $\mathrm{H}$ & $\mathrm{A}$ & Aug.-Feb. & Th & - & No & $\mathrm{NE}$ \\
\hline Sida cordifolia $\mathrm{L}$. & $\mathrm{S}$ & A & Aug.-Dec. & Th & - & No & $\mathrm{NE}$ \\
\hline Triumfetta rhomboidea Jacq. & $\mathrm{S}$ & $\mathrm{A}$ & Sep.-Jan. & $\mathrm{Th}$ & - & $\mathrm{Me}$ & $\mathrm{NE}$ \\
\hline Name of the species & Habit & Life-span & $\begin{array}{l}\text { Fl. and Fr. } \\
\text { time }\end{array}$ & $\begin{array}{l}\text { Raunkiaer's } \\
\text { life-form }\end{array}$ & Sub-type & $\begin{array}{c}\text { Leaf } \\
\text { spectra }\end{array}$ & $\begin{array}{l}\text { IUCN red list } \\
\text { status }\end{array}$ \\
\hline Urena sinuata $\mathrm{L}$. & $\mathrm{S}$ & $\mathrm{A}$ & Sep.-Dec. & $\mathrm{Ch}$ & - & No & $\mathrm{NE}$ \\
\hline \multicolumn{8}{|l|}{ Bixaceae Kunth } \\
\hline Bixa orellana L. & $\mathrm{T}$ & $\mathrm{P}$ & Oct.-Mar. & $\mathrm{Ph}$ & $\mathrm{N}$ & $\mathrm{Me}$ & $\mathrm{LC}$ \\
\hline \multicolumn{8}{|l|}{ Brassicales Bromhead } \\
\hline \multicolumn{8}{|l|}{ Capparaceae Juss. } \\
\hline Capparis zeylanica $\mathrm{L}$. & $\mathrm{C}$ & $\mathrm{P}$ & Mar.-Oct. & $\mathrm{Ph}$ & $\mathrm{M}$ & No & $\mathrm{NE}$ \\
\hline Crateva nurvala Buch.-Ham. & $\mathrm{T}$ & $\mathrm{P}$ & Mar.-Jul. & $\mathrm{Ph}$ & $\mathrm{M}$ & $\mathrm{Me}$ & $\mathrm{NE}$ \\
\hline \multicolumn{8}{|l|}{ Cleomaceae Bercht. and J. Presl } \\
\hline Cleome gynandra L. & $\mathrm{H}$ & A & Jul.-Sep. & Th & - & No & $\mathrm{NE}$ \\
\hline Cleome viscosa $\mathrm{L}$. & $\mathrm{H}$ & A & Sep.-Apr. & Th & - & No & $\mathrm{NE}$ \\
\hline \multicolumn{8}{|l|}{ SUPERASTERIDS } \\
\hline \multicolumn{8}{|l|}{ Santalales R. Br. ex Bercht. and J. Presl } \\
\hline \multicolumn{8}{|l|}{ Santalaceae R. Br. } \\
\hline Viscum cruciatum Sieber ex Boiss. & $\mathrm{S}$ & $\mathrm{P}$ & Jan.-Jun. & $\mathrm{Ph}$ & $\mathrm{N}$ & Le & $\mathrm{NE}$ \\
\hline \multicolumn{8}{|l|}{ Loranthaceae Juss. } \\
\hline Loranthus cordifolius Wall. & $\mathrm{S}$ & A & Jul.-Nov. & $\mathrm{Ph}$ & $\mathrm{N}$ & No & $\mathrm{NE}$ \\
\hline Scurrula atropurpurea (Blume) Danser & $\mathrm{S}$ & A & Nov.-Mar. & $\mathrm{Ph}$ & $\mathrm{N}$ & No & $\mathrm{NE}$ \\
\hline \multicolumn{8}{|c|}{ Caryophyllales Juss. ex Bercht. and J. Presl } \\
\hline \multicolumn{8}{|l|}{ Droseraceae Salisb. } \\
\hline Drosera burmanni Vahl & $\mathrm{H}$ & A & Nov.-Apr. & Th & - & Le & LC \\
\hline \multicolumn{8}{|l|}{ Amaranthaceae Juss } \\
\hline Achyranthes aspera L. & $\mathrm{H}$ & A & Sep.-Feb. & $\mathrm{Th}$ & - & $\mathrm{Mi}$ & $\mathrm{NE}$ \\
\hline Aerva lanata (L.) Juss. & $\mathrm{H}$ & $\mathrm{A}$ & Nov.-Jan. & Th & - & Le & $\mathrm{NE}$ \\
\hline Alternanthera sessilis (L.) R. Br. ex DC. & $\mathrm{H}$ & A & Jul.-Feb. & $\mathrm{Th}$ & - & $\mathrm{Mi}$ & $\mathrm{LC}$ \\
\hline Amaranthus spinosus L. & $\mathrm{H}$ & $\mathrm{A}$ & All & $\mathrm{Th}$ & - & $\mathrm{Na}$ & $\mathrm{NE}$ \\
\hline Amaranthus viridis $\mathrm{L}$. & $\mathrm{H}$ & A & All & $\mathrm{Th}$ & - & $\mathrm{Na}$ & $\mathrm{NE}$ \\
\hline Celosia argentea $\mathrm{L}$. & $\mathrm{H}$ & A & Sep.-Feb. & $\mathrm{Th}$ & - & $\mathrm{Na}$ & $\mathrm{NE}$ \\
\hline \multicolumn{8}{|l|}{ Aizoaceae Martinov } \\
\hline Trianthema portulaccastrum L. & $\mathrm{H}$ & A & Apr.-Oct. & $\mathrm{Th}$ & - & $\mathrm{Mi}$ & $\mathrm{NE}$ \\
\hline \multicolumn{8}{|l|}{ Nyctaginaceae Juss. } \\
\hline Boerhavia diffusa L. & $\mathrm{H}$ & $\mathrm{A}$ & Jun.-Dec. & $\mathrm{Th}$ & - & $\mathrm{Mi}$ & $\mathrm{NE}$ \\
\hline
\end{tabular}




\begin{tabular}{|c|c|c|c|c|c|c|c|}
\hline Portulacaceae Juss. & & & & & & & \\
\hline Portulaca oleracea $\mathrm{L}$. & $\mathrm{H}$ & $\mathrm{A}$ & Jun.-Dec. & Th & - & $\mathrm{Mi}$ & $\mathrm{NE}$ \\
\hline \multicolumn{8}{|l|}{ Cactaceae Juss. } \\
\hline Cereus pterogonus Lam. & $\mathrm{S}$ & $\mathrm{P}$ & Jun.-Jul. & $\mathrm{Ch}$ & - & $\mathrm{Le}$ & $\mathrm{NE}$ \\
\hline Opuntia stricta (Haw.) Haw. & $\mathrm{S}$ & $\mathrm{P}$ & Apr.-Aug. & $\mathrm{Ch}$ & - & Le & $\mathrm{LC}$ \\
\hline \multicolumn{8}{|l|}{ ASTERIDS } \\
\hline Name of the species & Habit & Life-span & $\begin{array}{l}\text { Fl. and Fr. } \\
\text { time }\end{array}$ & $\begin{array}{l}\text { Raunkiaer's } \\
\text { life-form }\end{array}$ & Sub-type & $\begin{array}{c}\text { Leaf } \\
\text { spectra }\end{array}$ & $\begin{array}{l}\text { IUCN red list } \\
\text { status }\end{array}$ \\
\hline \multicolumn{8}{|l|}{ Cornales Link } \\
\hline \multicolumn{8}{|l|}{ Cornaceae Bercht. and J. Presl } \\
\hline Alangium salviifolium (L. f.) Wangerin & $\mathrm{T}$ & $\mathrm{P}$ & Mar.-Jul. & $\mathrm{Ph}$ & $\mathrm{N}$ & $\mathrm{Me}$ & $\mathrm{NE}$ \\
\hline \multicolumn{8}{|l|}{ Ericales Bercht. and J. Presl } \\
\hline \multicolumn{8}{|l|}{ Lecythidaceae A. Rich. } \\
\hline Careya arborea Roxb. & $\mathrm{T}$ & $\mathrm{P}$ & Jan.-Apr. & $\mathrm{Ph}$ & $\mathrm{M}$ & $\mathrm{Mg}$ & $\mathrm{NE}$ \\
\hline \multicolumn{8}{|l|}{ Sapotaceae Juss. } \\
\hline $\begin{array}{l}\text { Madhuca longifolia var latifolia (Roxb.) A. } \\
\text { Chev. }\end{array}$ & $\mathrm{T}$ & $\mathrm{P}$ & Mar.-Jul. & $\mathrm{Ph}$ & MM & $\mathrm{Ma}$ & NE \\
\hline Mimusops elengi L. & $\mathrm{T}$ & $\mathrm{P}$ & Apr.-Sep. & $\mathrm{Ph}$ & MM & $\mathrm{Me}$ & $\mathrm{LC}$ \\
\hline \multicolumn{8}{|l|}{ Ebenaceae Gurke } \\
\hline Diospyros melanoxylon Roxb. & $\mathrm{T}$ & $\mathrm{P}$ & Apr.-Jul. & $\mathrm{Ph}$ & MM & $\mathrm{Ma}$ & $\mathrm{NE}$ \\
\hline \multicolumn{8}{|l|}{ Gentianales Juss. ex Bercht. and J. Presl } \\
\hline \multicolumn{8}{|l|}{ Rubiaceae Juss. } \\
\hline Gardenia gummifera L. f. & $\mathrm{T}$ & $\mathrm{P}$ & Mar.-Aug. & $\mathrm{Ph}$ & $\mathrm{N}$ & No & $\mathrm{LC}$ \\
\hline Gardenia resinifera Roth & $\mathrm{S}$ & $\mathrm{P}$ & Feb.-Jun. & $\mathrm{Ph}$ & $\mathrm{N}$ & No & $\mathrm{NE}$ \\
\hline Haldina cordifolia (Roxb.) Ridsdale & $\mathrm{T}$ & $\mathrm{P}$ & Jun.-Dec. & $\mathrm{Ph}$ & $\mathrm{MM}$ & $\mathrm{Ma}$ & $\mathrm{NE}$ \\
\hline Hedyotis neesiana Arn. & $\mathrm{H}$ & A & Jun.-Nov. & Th & - & $\mathrm{Na}$ & $\mathrm{NE}$ \\
\hline Meyna spinose Roxb. ex Link & $\mathrm{S}$ & $\mathrm{P}$ & Mar.-Jun. & $\mathrm{Ch}$ & - & $\mathrm{Me}$ & $\mathrm{NE}$ \\
\hline Morinda citrifolia $\mathrm{L}$. & $\mathrm{T}$ & $\mathrm{P}$ & Feb.-May & $\mathrm{Ph}$ & $\mathrm{N}$ & $\mathrm{Ma}$ & $\mathrm{NE}$ \\
\hline Neolamarckia cadamba (Roxb.) Bosser & $\mathrm{T}$ & $\mathrm{P}$ & Jul.-Nov. & $\mathrm{Ph}$ & MM & $\mathrm{Ma}$ & NE \\
\hline Oldenlandia corymbosa $\mathrm{L}$. & $\mathrm{H}$ & $\mathrm{A}$ & Aug.-Feb. & Th & - & Le & $\mathrm{LC}$ \\
\hline Spermacoce articularis L. f. & $\mathrm{H}$ & A & All & Th & - & $\mathrm{Na}$ & $\mathrm{NE}$ \\
\hline \multicolumn{8}{|l|}{ Loganiaceae R.Br. ex Mart. } \\
\hline Strychnos nux-vomica L. & $\mathrm{T}$ & $\mathrm{P}$ & Mar.-Jan. & $\mathrm{Ph}$ & MM & $\mathrm{Me}$ & $\mathrm{NE}$ \\
\hline \multicolumn{8}{|l|}{ Apocynaceae Juss. } \\
\hline Alstonia scholaris (L.) R. Br. & $\mathrm{T}$ & $\mathrm{P}$ & Nov.-Aug. & $\mathrm{Ph}$ & $\mathrm{MM}$ & $\mathrm{Me}$ & $\mathrm{LC}$ \\
\hline Calotropis gigantean (L.) Dryand. & $\mathrm{S}$ & $\mathrm{P}$ & Mar.-Feb. & $\mathrm{Ch}$ & - & $\mathrm{Ma}$ & $\mathrm{NE}$ \\
\hline Carissa spinarum A. DC. & $\mathrm{C}$ & $\mathrm{P}$ & Mar.-Oct. & $\mathrm{Ph}$ & $\mathrm{N}$ & No & $\mathrm{NE}$ \\
\hline Cascabela thevetia (L.) Lippold & $\mathrm{T}$ & $\mathrm{P}$ & All & $\mathrm{Ph}$ & $\mathrm{N}$ & $\mathrm{Mi}$ & $\mathrm{LC}$ \\
\hline Catharanthus roseus (L.) G. Don. & $\mathrm{S}$ & $\mathrm{P}$ & All & Th & - & No & $\mathrm{NE}$ \\
\hline
\end{tabular}


Sen and Bhakat/ Journal of Tropical Forestry and Environment Vol. 10 No. 01 (2020) 17-39

\begin{tabular}{|c|c|c|c|c|c|c|c|}
\hline Cryptolepis dubia (Burm.f.) M.R.Almeida & $\mathrm{C}$ & $\mathrm{P}$ & Apr.-Mar. & $\mathrm{Ph}$ & $\mathrm{N}$ & No & $\mathrm{NE}$ \\
\hline Gymnema sylvestre (Retz.) R.Br. ex Sm. & $\mathrm{C}$ & $\mathrm{P}$ & Apr.-Mar. & $\mathrm{Ph}$ & $\mathrm{N}$ & $\mathrm{Mi}$ & $\mathrm{NE}$ \\
\hline Hemidesmus indicus (L.) R. Br. ex Schult. & $\mathrm{C}$ & $\mathrm{P}$ & Aug.-Jan. & $\mathrm{Ph}$ & $\mathrm{N}$ & $\mathrm{Mi}$ & $\mathrm{NE}$ \\
\hline Holarrhena pubescens Wall. ex G. Don & $\mathrm{T}$ & $\mathrm{P}$ & Apr.-Feb. & $\mathrm{Ph}$ & $\mathrm{N}$ & $\mathrm{Ma}$ & $\mathrm{LC}$ \\
\hline Ichnocarpus frutescens (L.) W. T. Aiton & $\mathrm{S}$ & $\mathrm{P}$ & Sep.-Mar. & $\mathrm{Ph}$ & $\mathrm{N}$ & No & NE \\
\hline Marsdenia sylvestris (Retz.) P. 1. Forst. & $\mathrm{C}$ & $\mathrm{P}$ & Apr.-Mar. & $\mathrm{Ph}$ & $\mathrm{N}$ & Mi & $\mathrm{NE}$ \\
\hline Nerium oleander $($ L.) & $\mathrm{S}$ & $\mathrm{P}$ & Jul.-Apr. & $\mathrm{Ph}$ & $\mathrm{N}$ & No & $\mathrm{LC}$ \\
\hline Name of the species & Habit & Life-span & $\begin{array}{l}\text { Fl. and Fr. } \\
\text { time }\end{array}$ & $\begin{array}{c}\text { Raunkiaer's } \\
\text { life-form }\end{array}$ & Sub-type & $\begin{array}{c}\text { Leaf } \\
\text { spectra }\end{array}$ & $\begin{array}{c}\text { IUCN red list } \\
\text { status }\end{array}$ \\
\hline Pergularia daemia (Forssk.) Chiov. & $\mathrm{C}$ & $\mathrm{P}$ & Sep.-Jan. & $\mathrm{Ph}$ & $\mathrm{N}$ & $\mathrm{Me}$ & $\mathrm{NE}$ \\
\hline Plumeria rubra (L.) & $\mathrm{T}$ & $\mathrm{P}$ & Oct.-May & $\mathrm{Ph}$ & M & No & $\mathrm{LC}$ \\
\hline Rauvolfia serpentina (L.) Benth. ex Kurz & $\mathrm{S}$ & $\mathrm{P}$ & Mar.-Dec. & Th & - & $\mathrm{Me}$ & $\mathrm{NE}$ \\
\hline Rauvolfia tetraphylla $\mathrm{L}$. & $\mathrm{S}$ & $\mathrm{P}$ & Feb.-Dec. & $\mathrm{Ch}$ & - & No & $\mathrm{NE}$ \\
\hline Tylophora indica (Burm. f.) Merr. & $\mathrm{C}$ & A & Apr.-Oct. & $\mathrm{Ph}$ & $\mathrm{N}$ & No & $\mathrm{NE}$ \\
\hline Vallaris solanacea (Roth) Kuntze & $\mathrm{C}$ & $\mathrm{P}$ & Apr.-Jan. & $\mathrm{Ph}$ & $\mathrm{N}$ & $\mathrm{Me}$ & $\mathrm{NE}$ \\
\hline \multicolumn{8}{|l|}{ Boraginales Juss. ex Bercht. and J. Presl } \\
\hline \multicolumn{8}{|l|}{ Boraginaceae Juss. } \\
\hline Heliotropium indicum $\mathrm{L}$. & $\mathrm{H}$ & $\mathrm{A}$ & Oct.-Jan. & Th & - & No & $\mathrm{NE}$ \\
\hline \multicolumn{8}{|l|}{ Solanales Juss. ex Bercht. and J. Presl } \\
\hline \multicolumn{8}{|l|}{ Convolvulaceae Juss. } \\
\hline Cuscuta reflexa Roxb. & $\mathrm{C}$ & $\mathrm{P}$ & Nov.-Mar. & $\mathrm{Ph}$ & $\mathrm{N}$ & Ap & $\mathrm{NE}$ \\
\hline Evolvulus alsinoides (L.) L. & $\mathrm{H}$ & $\mathrm{A}$ & Jul.-Feb. & Th & - & $\mathrm{Na}$ & $\mathrm{NE}$ \\
\hline Ipomoea aquatica Forssk. & $\mathrm{H}$ & $\mathrm{P}$ & All & $\mathrm{Th}$ & - & No & $\mathrm{LC}$ \\
\hline Ipomoea mauritiana Jacq, & $\mathrm{C}$ & $\mathrm{P}$ & Aug.-Dec. & $\mathrm{Ph}$ & $\mathrm{N}$ & $\mathrm{Ma}$ & $\mathrm{NE}$ \\
\hline Jacquemontia paniculata (Burm. f.) Hallier f. & $\mathrm{T}$ & $\mathrm{P}$ & Sep.-Jan. & $\mathrm{Ph}$ & $\mathrm{N}$ & $\mathrm{Mi}$ & $\mathrm{NE}$ \\
\hline Rivea hypocrateriformis Choisy & $\mathrm{C}$ & $\mathrm{P}$ & Aug.-Oct. & $\mathrm{Ph}$ & $\mathrm{N}$ & No & $\mathrm{NE}$ \\
\hline \multicolumn{8}{|l|}{ Solanaceae Juss. } \\
\hline Datura metel L. & $\mathrm{S}$ & $\mathrm{P}$ & Aug.-May & $\mathrm{Ch}$ & - & $\mathrm{Ma}$ & $\mathrm{NE}$ \\
\hline Datura stramonium L. & $\mathrm{S}$ & $\mathrm{P}$ & Jul.-Oct. & $\mathrm{Ch}$ & - & $\mathrm{Ma}$ & $\mathrm{NE}$ \\
\hline Physalis minima $\mathrm{L}$. & $\mathrm{H}$ & A & Aug.-Dec. & $\mathrm{Ch}$ & - & $\mathrm{Ma}$ & $\mathrm{NE}$ \\
\hline Solanum americanum Mill. & $\mathrm{H}$ & A & Dec.-Jun. & Th & - & $\mathrm{Ma}$ & $\mathrm{NE}$ \\
\hline Solanum rudepannum Dunal & $\mathrm{H}$ & A & Dec.-Jun. & Th & - & $\mathrm{Ma}$ & $\mathrm{LC}$ \\
\hline Solanum surattense Burm. f. & $\mathrm{H}$ & A & Aug.-Dec. & Th & - & $\mathrm{Ma}$ & $\mathrm{NE}$ \\
\hline Solanum torvum Sw. & $\mathrm{H}$ & $\mathrm{P}$ & Jul.-Mar. & $\mathrm{Ch}$ & - & Ma & NE \\
\hline Solanum virginianum L. & $\mathrm{H}$ & A & Dec.-Jun. & $\mathrm{Th}$ & - & Ma & NE \\
\hline \multicolumn{8}{|l|}{ Lamiales Bromhead } \\
\hline \multicolumn{8}{|l|}{ Oleaceae Hoffmanns. and Link } \\
\hline Nyctanthes arbor-tristis L. & $\mathrm{T}$ & $\mathrm{P}$ & Sep.-Jan. & $\mathrm{Ch}$ & - & Mi & $\mathrm{NE}$ \\
\hline Plantaginaceae Juss. & & & & & & & \\
\hline
\end{tabular}




\begin{tabular}{|c|c|c|c|c|c|c|c|}
\hline Bacopa monnieri (L.) Pennell & $\mathrm{H}$ & A & Apr.-Jan. & Th & - & $\mathrm{Na}$ & $\mathrm{LC}$ \\
\hline Limnophila indica (L.) Druce & $\mathrm{H}$ & A & Sep.-Jan. & Th & - & $\mathrm{Na}$ & $\mathrm{LC}$ \\
\hline Scoparia dulcis L. & $\mathrm{H}$ & A & May-Dec. & Th & - & $\mathrm{Na}$ & $\mathrm{NE}$ \\
\hline \multicolumn{8}{|l|}{ Martyniaceae Horan. } \\
\hline Martynia aпnиa L. & $\mathrm{H}$ & A & Aug.-Dec. & $\mathrm{Ch}$ & - & $\mathrm{Me}$ & $\mathrm{NE}$ \\
\hline \multicolumn{8}{|l|}{ Acanthaceae Juss. } \\
\hline Andrographis echioides (L.) Nees & $\mathrm{H}$ & $\mathrm{A}$ & Jul.-Oct. & Th & - & No & $\mathrm{NE}$ \\
\hline Andrographis paniculata (Burm. f.) Nees & $\mathrm{H}$ & $\mathrm{A}$ & Sep.-Apr. & Th & - & No & $\mathrm{NE}$ \\
\hline Name of the species & Habit & Life-span & $\begin{array}{l}\text { Fl. and Fr. } \\
\text { time }\end{array}$ & $\begin{array}{c}\text { Raunkiaer's } \\
\text { life-form }\end{array}$ & Sub-type & $\begin{array}{c}\text { Leaf } \\
\text { spectra }\end{array}$ & $\begin{array}{c}\text { IUCN red list } \\
\text { status }\end{array}$ \\
\hline Barleria lupulina Lindl. & $\mathrm{S}$ & $\mathrm{P}$ & Dec.-Apr. & $\mathrm{Ch}$ & - & $\mathrm{Mi}$ & $\mathrm{NE}$ \\
\hline Barleria prionitis (L.) & $\mathrm{S}$ & $\mathrm{P}$ & Dec.-Apr. & $\mathrm{Ch}$ & - & $\mathrm{Mi}$ & $\mathrm{NE}$ \\
\hline Dicliptera bupleuroides Nees & $\mathrm{H}$ & A & Jun.-Oct. & Th & - & No & NE \\
\hline Ecbolium viride (Forsk.) Alston & $\mathrm{H}$ & $\mathrm{P}$ & Dec.-Apr. & $\mathrm{Ch}$ & - & $\mathrm{Mi}$ & $\mathrm{NE}$ \\
\hline Hemigraphis hirta T. Anders. & $\mathrm{H}$ & A & Aug.-Nov. & Th & - & $\mathrm{Mi}$ & $\mathrm{NE}$ \\
\hline Hygrophila auriculata (Schumach.) Heine & $\mathrm{H}$ & A & Sep.-Jan. & Th & - & $\mathrm{Mi}$ & $\mathrm{LC}$ \\
\hline Justicia adhatoda L. & $\mathrm{S}$ & $\mathrm{P}$ & Feb.-Apr. & $\mathrm{Ch}$ & - & $\mathrm{Me}$ & $\mathrm{NE}$ \\
\hline Justicia gendarussa Burm.f. & $\mathrm{S}$ & $\mathrm{P}$ & Feb.-Apr. & $\mathrm{Ch}$ & - & $\mathrm{Me}$ & $\mathrm{NE}$ \\
\hline Ruellia tuberosa L. & $\mathrm{H}$ & A & Aug.-Nov. & Th & - & $\mathrm{Mi}$ & $\mathrm{NE}$ \\
\hline Rungia pectinata (L.) Nees & $\mathrm{H}$ & A & All & Th & - & Mi & $\mathrm{NE}$ \\
\hline \multicolumn{8}{|l|}{ Bignoniaceae Juss. } \\
\hline Tecoma stans (L.) Juss. ex Kunth & $\mathrm{T}$ & $\mathrm{P}$ & Nov.-Mar. & $\mathrm{Ph}$ & $\mathrm{N}$ & $\mathrm{Me}$ & $\mathrm{NE}$ \\
\hline Stereospermum chelonoides (L. f.) DC. & $\mathrm{T}$ & $\mathrm{P}$ & Aug.-Feb. & $\mathrm{Ph}$ & $\mathrm{M}$ & $\mathrm{Me}$ & $\mathrm{NE}$ \\
\hline \multicolumn{8}{|l|}{ Verbenaceae J. St. Hil. } \\
\hline Lantana camara (L.) & $\mathrm{S}$ & $\mathrm{P}$ & Nov.-Feb. & $\mathrm{Ch}$ & - & No & $\mathrm{NE}$ \\
\hline Lippia javanica (Burm. f.) Spreng. & $\mathrm{S}$ & $\mathrm{P}$ & Sep.-Apr. & $\mathrm{Ch}$ & - & $\mathrm{Mi}$ & $\mathrm{NE}$ \\
\hline \multicolumn{8}{|l|}{ Lamiaceae Martinov } \\
\hline Anisochilus carnosus (L. f.) Wall. & $\mathrm{S}$ & A & Sep.-Jan. & $\mathrm{Ch}$ & - & No & $\mathrm{NE}$ \\
\hline Anisomeles indica (L.) Kuntze & $\mathrm{H}$ & A & Sep.-Jan. & $\mathrm{Ch}$ & - & Mi & NE \\
\hline Clerodendrum infortunatum L. & $\mathrm{S}$ & $\mathrm{P}$ & Feb.-Jul. & $\mathrm{Ch}$ & - & $\mathrm{Ma}$ & $\mathrm{NE}$ \\
\hline Hyptis suaveolens (L.) Poit. & $\mathrm{S}$ & A & Sep.-Jan. & $\mathrm{Ch}$ & - & $\mathrm{Me}$ & $\mathrm{NE}$ \\
\hline Leonotis nepetifolia (L.) R. Br. & $\mathrm{S}$ & A & Apr.-Jul. & Th & - & $\mathrm{Me}$ & $\mathrm{NE}$ \\
\hline Leonurus sibiricus L. & $\mathrm{S}$ & A & Sep.-Feb. & $\mathrm{Ch}$ & - & Mi & $\mathrm{NE}$ \\
\hline Leucas cephalotes (Roth) Spreng. & $\mathrm{H}$ & A & Sep.-Dec. & Th & - & $\mathrm{Mi}$ & $\mathrm{NE}$ \\
\hline Mentha longifolia (L.) L. & $\mathrm{S}$ & A & Apr.-Jul. & $\mathrm{Th}$ & - & $\mathrm{Mi}$ & $\mathrm{LC}$ \\
\hline Ocimum americanum $\mathrm{L}$. & $\mathrm{S}$ & $\mathrm{P}$ & All & $\mathrm{Ch}$ & - & $\mathrm{Na}$ & NE \\
\hline Ocimum basilicum L. & $\mathrm{H}$ & $\mathrm{P}$ & May-Jul. & $\mathrm{Ch}$ & - & $\mathrm{Na}$ & $\mathrm{NE}$ \\
\hline Ocimum tenuiflorum $\mathrm{L}$. & $\mathrm{S}$ & $\mathrm{P}$ & Aug.-Jan. & $\mathrm{Ch}$ & - & $\mathrm{Na}$ & NE \\
\hline Plectranthus amboinicus (Lour.) Spreng. & $\mathrm{H}$ & A & May-Sep. & Th & - & $\mathrm{Mi}$ & $\mathrm{NE}$ \\
\hline
\end{tabular}


Sen and Bhakat/ Journal of Tropical Forestry and Environment Vol. 10 No. 01 (2020) 17-39

\begin{tabular}{|c|c|c|c|c|c|c|c|}
\hline Premna mollissima Roth & $\mathrm{T}$ & $\mathrm{P}$ & May-Jul. & $\mathrm{Ph}$ & M & No & $\mathrm{NE}$ \\
\hline Tectona grandis L. f. & $\mathrm{T}$ & $\mathrm{P}$ & Jul.-Jan. & $\mathrm{Ph}$ & MM & $\mathrm{Mg}$ & $\mathrm{NE}$ \\
\hline Vitex negundo L. & $\mathrm{T}$ & $\mathrm{P}$ & Mar.-Jun. & $\mathrm{Ph}$ & $\mathrm{N}$ & $\mathrm{Mi}$ & $\mathrm{LC}$ \\
\hline \multicolumn{8}{|l|}{ Asterales Link } \\
\hline \multicolumn{8}{|l|}{ Asteraceae Bercht. and J.Presl } \\
\hline Ageratum conyzoides (L.) L. & $\mathrm{H}$ & A & Nov.-Mar. & Th & - & $\mathrm{Mi}$ & $\mathrm{LC}$ \\
\hline $\begin{array}{l}\text { Ayapana triplinervis (Vahl) R. M. King and } \mathrm{H} \text {. } \\
\text { Rob. }\end{array}$ & $\mathrm{H}$ & A & Aug.-Dec. & Th & - & $\mathrm{Mi}$ & $\mathrm{NE}$ \\
\hline Name of the species & Habit & Life-span & $\begin{array}{l}\text { Fl. and Fr. } \\
\text { time }\end{array}$ & $\begin{array}{l}\text { Raunkiaer's } \\
\text { life-form }\end{array}$ & Sub-type & $\begin{array}{c}\text { Leaf } \\
\text { spectra }\end{array}$ & $\begin{array}{l}\text { IUCN red list } \\
\text { status }\end{array}$ \\
\hline Baccharoides anthelmintica (L.) Moench & $\mathrm{H}$ & A & Sep.-Mar. & $\mathrm{Th}$ & - & $\mathrm{Me}$ & $\mathrm{NE}$ \\
\hline Blumea lacera (Burm.f.) DC. & $\mathrm{H}$ & A & Aug.-Feb. & $\mathrm{Th}$ & - & $\mathrm{Mi}$ & $\mathrm{NE}$ \\
\hline $\begin{array}{l}\text { Chromolaena odorata (L.) R. M. King and H. } \\
\text { Rob. }\end{array}$ & $S$ & A & Mar.-Sep. & $\mathrm{Ch}$ & - & $\mathrm{Mi}$ & $\mathrm{NE}$ \\
\hline Cyanthillium albicans (DC.) H. Rob. & $\mathrm{H}$ & $\mathrm{A}$ & Aug.-Mar. & Th & - & $\mathrm{Mi}$ & $\mathrm{NE}$ \\
\hline Cyanthillium cinereum (L.) H. Rob. & $\mathrm{H}$ & $\mathrm{A}$ & Nov.-Feb. & $\mathrm{Th}$ & - & $\mathrm{Me}$ & $\mathrm{NE}$ \\
\hline Eclipta prostrata (L.) L. & $\mathrm{H}$ & $\mathrm{A}$ & All & Th & - & $\mathrm{Mi}$ & $\mathrm{LC}$ \\
\hline Elephantopus scaber L. & $\mathrm{H}$ & $\mathrm{A}$ & Sep.-Jan. & $\mathrm{Th}$ & - & No & $\mathrm{NE}$ \\
\hline Enydra fluctuans DC. & $\mathrm{H}$ & $\mathrm{A}$ & Dec.-Mar. & Th & - & $\mathrm{Mi}$ & $\mathrm{LC}$ \\
\hline Grangea maderaspatana (L.) Poir. & $\mathrm{H}$ & $\mathrm{A}$ & Dec.-May & Th & - & Le & $\mathrm{LC}$ \\
\hline Sonchus oleraceus (L.) L. & $\mathrm{H}$ & $\mathrm{A}$ & Sep.-Jan. & Th & - & $\mathrm{Na}$ & $\mathrm{NE}$ \\
\hline Sphaeranthus senegalensis DC. & $\mathrm{H}$ & A & Nov.-Apr. & Th & - & $\mathrm{Le}$ & $\mathrm{LC}$ \\
\hline Sphagneticola calendulacea (L.) Pruski & $\mathrm{H}$ & $\mathrm{A}$ & All & Th & - & $\mathrm{Me}$ & $\mathrm{NE}$ \\
\hline Synedrella nodiflora (L.) Gaertn. & $\mathrm{H}$ & $\mathrm{A}$ & Sep.-Jan. & Th & - & No & $\mathrm{NE}$ \\
\hline Tridax procumbens (L.) L. & $\mathrm{H}$ & A & All & Th & - & $\mathrm{Na}$ & $\mathrm{NE}$ \\
\hline Xanthium strumarium L. & $\mathrm{H}$ & $\mathrm{A}$ & Sep.-Apr. & $\mathrm{Th}$ & - & $\mathrm{Me}$ & $\mathrm{NE}$ \\
\hline \multicolumn{8}{|l|}{ Apiales Nakai } \\
\hline \multicolumn{8}{|l|}{ Apiaceae Lindl. } \\
\hline Centella asiatica (L.) Urb. & $\mathrm{H}$ & $\mathrm{A}$ & Jul.-Jan. & Th & - & No & $\mathrm{LC}$ \\
\hline
\end{tabular}

\section{Abbreviation}

In Habit: C-Climber; H-Herb; S-Shrub; T-Tree

In Life-span: A-Annual; B-Biennial; P-Perennial

In Flowering and fruiting time: Jan.-January; Feb.-February; Mar.-March; Apr.-April; Jun.-June; Jul.-July; Aug.-August; Sep.-September; Oct.-October; Nov.-

November; Dec.-December; All-All season

In Raunkiaer's life-form and Sub-type: Ch-Chamaephytes; Cr-Cryptophytes; He-Hemicryptophytes; M-Mesophanerophyte; MM-Megaphanerophytes; NNanophanerophytes; Ph-Phanerophytes; Th-Therophytes

In Leaf spectra: Ap-Aphyllous; Le-Leptophyll; Na-Nanophyll; Mi-Microphyll; No-Notophyll; Me-Mesophyll; Ma-Macrophyll; Mg-Megaphyll

In IUCN red list status: DD-Data Deficient; LC-Least Concern; NE-Not Evaluated; VU-Vulnerable; NT-Near Threatened 
Table 2: Total angiospermic taxa.

\begin{tabular}{lcccccccc}
\hline \multirow{2}{*}{ Group } & \multirow{2}{*}{ Orders } & \multirow{2}{*}{ Families } & \multirow{2}{*}{ Genera } & \multicolumn{5}{c}{ Species } \\
\cline { 5 - 9 } & & & & Herbs & Shrubs & Trees & Climber & Total \\
\hline Dicots & 27 & 62 & 203 & 79 & 59 & 70 & 41 & 249 \\
Monocots & 9 & 17 & 46 & 40 & 4 & 6 & 8 & 58 \\
Total & 36 & 79 & 249 & 119 & 63 & 76 & 49 & 307 \\
\hline
\end{tabular}

\subsection{Life span}

In the sacred grove, 109 (35.50\%) annual plants would go through their life cycle in one growing season. As many as $1(0.33 \%)$ biennial plant whose life cycle spans two years. As many as $197(64.17 \%)$ perennial plants that could survive most unfavorable conditions and stayed alive for more than two years (Table 1).

\subsection{Life form and biological spectrum}

The biological spectrum shows that phanerophytes 128 (41.69\%) was the dominant, followed by therophytes 81 (26.38\%), chamaephytes 52 (16.95\%), hemicryptophytes $27(8.79 \%)$, and cryptophytes $19(6.19 \%)$. Of the phanerophytes, nanophanerophytes $73(23.78 \%)$ was dominant than mesophanerophytes $31(10.10 \%)$ and megaphanerophytes 24 (7.82\%) (Table 3, Figure 7). It reveals that therophytes, chamaephytes, and cryptophytes constituted the higher percentage $13.38 \%, 7.95 \%$ and $0.19 \%$ respectively than the normal spectrum exhibiting "thero-chamae-cryptophytic" phytoclimate.

Further, the number of hemicryptophytes (17.21\%) and phanerophytes $(4.31 \%)$ was comparatively smaller in percentage than the Raunkiaer's normal spectrum. Of the phanerophytes, nanophanerophytes (8.78\%) and megaphanerophytes (4.82\%) were somewhat larger and mesophanerophyte $(17.9 \%)$ was a comparatively smaller value than the Raunkiaer's normal spectrum (Table 3, Figure 7). This result was probably due to the local protection under certain taboos of the sacred grove. The dominant therophytes, chamaephytes, and cryptophytes altogether constituted $49.52 \%$ of the life forms proportion. Therophytes showed the maximum divergence of the normal spectrum; other workers had also reported a similar phytoclimatic association for different tracks of vegetation (Misra et al., 1979; Saxena et al., 1982; Rajendraprasad et al., 1998; Sen, 2018). The dominance of therophytes (81 species, $26.38 \%$ ) indicates that the investigated area was under mild biotic pressure (Sher et al., 2014). Many plant species were decreasing in the area. It would be the moral and ethical duty of the local people to protect the plant resources.

Table 3: Biological spectrum (\% of all life forms) of study site and its comparison with Raunkiaer's normal spectrum.

\begin{tabular}{lcccc}
\hline Life forms & $\begin{array}{c}\text { Total no. of } \\
\text { species }\end{array}$ & $\begin{array}{c}\text { Biological } \\
\text { spectrum (\%) } \\
\text { of the study site }\end{array}$ & $\begin{array}{c}\text { Raunkiaer's normal } \\
\text { spectrum (\%) }\end{array}$ & $\begin{array}{c}\text { Deviation=(Raunkiaer's } \\
\text { normal spectrum- } \\
\text { Biological spectrum) }\end{array}$ \\
\hline Phanerophytes (Ph) & 128 & 41.69 & 46.00 & -4.31 \\
Megaphanerophytes (MM) & 24 & 7.82 & 3.00 & 4.82 \\
Mesophanerophytes (M) & 31 & 10.10 & 28.00 & -17.90 \\
Nanophanerophytes (N) & 73 & 23.78 & 15.00 & 8.78 \\
Chamaephytes (Ch) & 52 & 16.95 & 9.00 & 7.95 \\
Hemicryptophytes (He) & 27 & 8.79 & 26.00 & -17.21 \\
Cryptophytes (Cr) & 19 & 6.19 & 6.00 & 0.19 \\
Therophytes (Th) & 81 & 26.38 & 13.00 & 13.38 \\
Total & 307 & 100.00 & 100.00 & \\
\hline
\end{tabular}




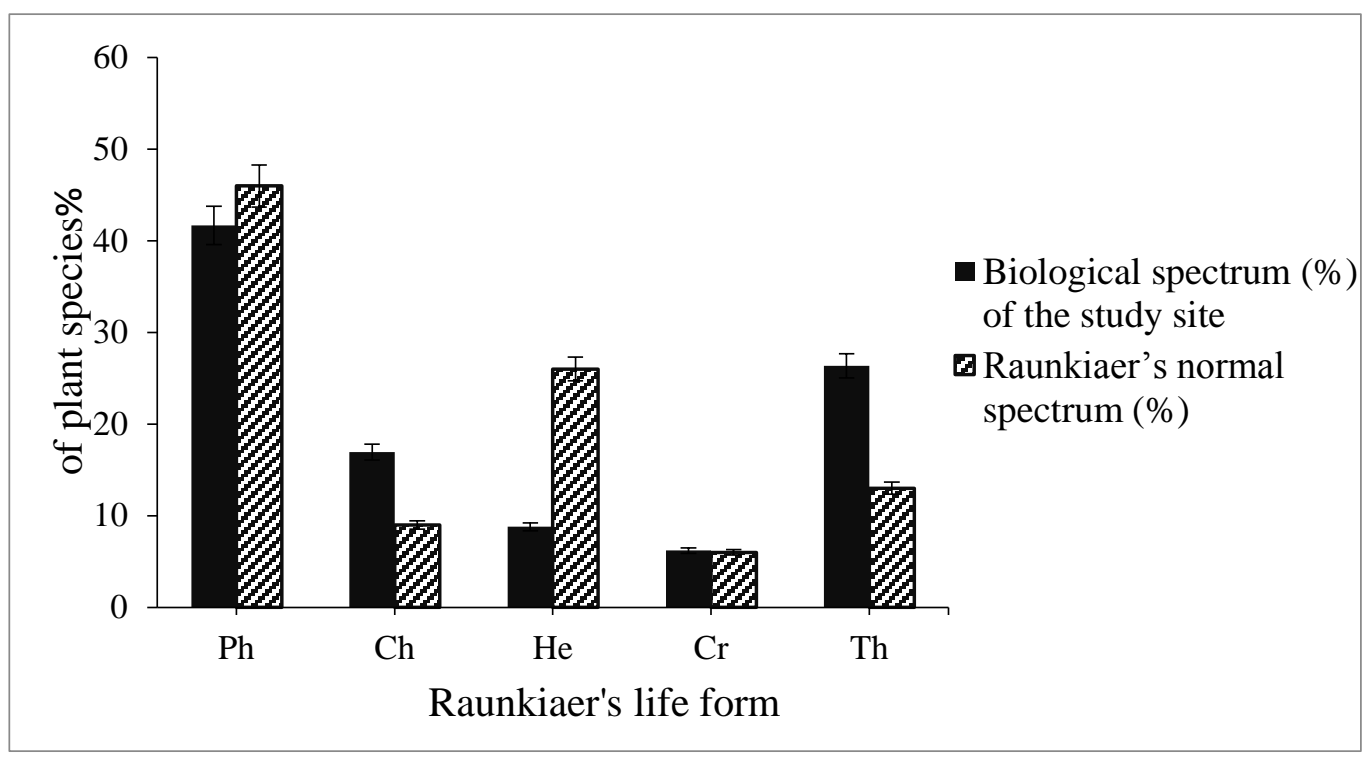

Figure 7. Comparison of biological spectrum with Raunkiaer's normal spectrum.

\subsection{Leaf size spectra}

The overall leaf size spectra showed that there were leptophyll 34 (11.11\%), nanophyll 42 (13.73\%), microphyll 50 (16.34\%), notophyll 52 (16.99\%), mesophyll 67 (21.90\%), macrophyll 49 (16.01\%) and megaphyll 12 (3.92\%). Cuscuta reflexa is only aphyllous species. As regards the leaf size spectra, mesophyll was found to be high followed by notophyll, microphyll, macrophyll, nanophyll, leptophyll, and megaphyll (Table 1, 4). In case of leaf spectra, the presence of leptophyll $19(6.21 \%)$, nanophyll 21 ( 6.86\%), microphyll 22 (7.19\%), notophyll 24 (7.84\%), mesophyll 43 (14.05\%), macrophyll 24 (7.84\%) and megaphyll 7 (2.29\%) have the maximum in comparison to hemicryptophytes, therophytes, therophytes, phanerophytes, phanerophytes, phanerophytes and phanerophytes respectively (Table 4). Cyperaceae 8 (2.61\%), Poaceae 5 (1.63\%), Fabaceae $9(2.93 \%)$, Apocynaceae 8 (2.61\%), Malvaceae 6 (1.95\%), Solanaceae $8(2.61 \%)$ and Araceae $3(0.98 \%)$ were dominant families of leptophyll, nanophyll, microphyll, notophyll, mesophyll, macrophyll and megaphyll respectively (Table 4, Figure 8). This result was also similar to other tropical forests in Asia (Bohman, 2004; Gillison, 2018).

Table 4: Life-form analysis with different leaf size.

\begin{tabular}{lcrrrrrrrr}
\hline Raunkiaer's & \multicolumn{8}{c}{ Leaf spectra } & \multirow{2}{*}{ Total } \\
\cline { 2 - 8 } life form & $\mathrm{Ap}$ & $\mathrm{Le}$ & $\mathrm{Na}$ & $\mathrm{Mi}$ & $\mathrm{No}$ & $\mathrm{Me}$ & $\mathrm{Ma}$ & $\mathrm{Mg}$ & \\
\hline Ph & 1 & 2 & 11 & 16 & 24 & 43 & 24 & 7 & 128 \\
$\mathrm{MM}$ & & & 1 & 2 & & 7 & 12 & 2 & 24 \\
$\mathrm{M}$ & & & 5 & 1 & 8 & 9 & 5 & 3 & 31 \\
$\mathrm{~N}$ & 1 & 2 & 5 & 13 & 16 & 27 & 7 & 2 & 73 \\
$\mathrm{Ch}$ & & 4 & 5 & 9 & 8 & 11 & 15 & & 52 \\
$\mathrm{He}$ & & 19 & 5 & 2 & 1 & & & & 27 \\
$\mathrm{Cr}$ & & 2 & & 1 & 2 & 4 & 5 & 5 & 19 \\
Th & & 7 & 21 & 22 & 17 & 9 & 5 & & 81 \\
Total & 1 & 34 & 42 & 50 & 52 & 67 & 49 & 12 & 307 \\
\hline
\end{tabular}

\section{Abbreviation}

In Raunkiaer's life-form: Ch-Chamaephytes; Cr-Cryptophytes; He-Hemicryptophytes; M-Mesophanerophyte; MMMegaphanerophytes; N-Nanophanerophytes; Ph-Phanerophytes; Th-Therophytes 
In Leaf spectra: Ap-Aphyllous; Le-Leptophyll; Na-Nanophyll; Mi-Microphyll; No-Notophyll; Me-Mesophyll; MaMacrophyll; Mg-Megaphyll

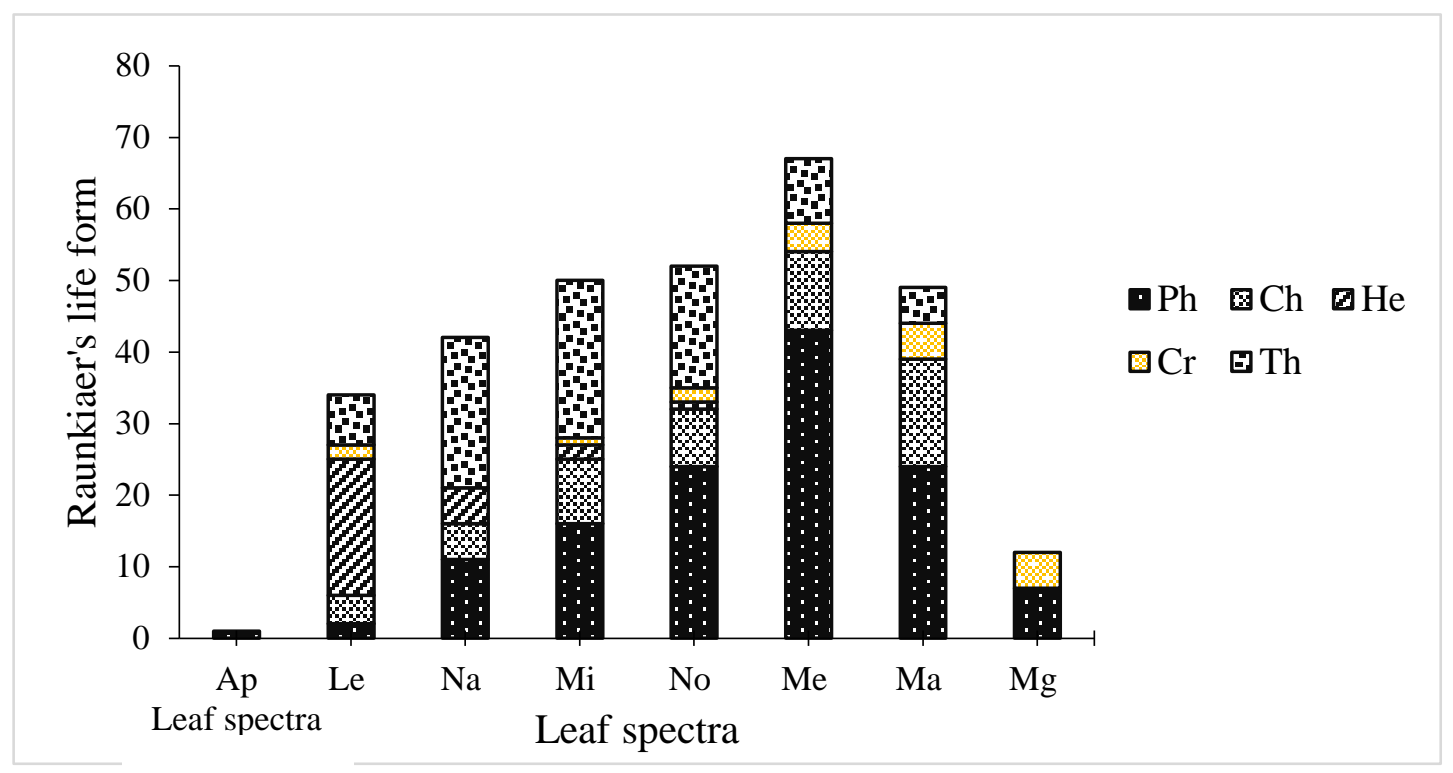

Figure. 8. Analysis of life form with different leaf size.

\subsection{Conservation status and IUCN categories}

Among these 307 plant species, 220 plants have not been evaluated still now. There were 85 least concerned (LC), 1 data deficient (DD), 1 near threatened (NT) species according to the IUCN (IUCN, 2020) (Table 1).

Based on the above-mentioned phytosociological analysis with ecological information on IUCN Red Listed plants, it is revealed that plants are still present and regenerate in the sacred grove but locally disappear in highly disturbed areas. This study would highlight the status and distribution of the species in the study area, the ecological characteristics necessary for their survival, and the threats to some of the species identified by following the IUCN (2020) criteria. It is because IUCN Red List applications are a global plant diversity barometer (Brummitt et al., 2008). Various factors caused the increase in numbers of threatened species in the area. Grasing was a major cause which led to the destruction of seedlings. The most critical factor that caused the decline of the endemic useful species was human activity, such as pilgrimage, dying of the plant and land use change.

\section{Conclusion}

The current study indicates the option of using Raunkiaer's strategy to ascertain the notable differences between the populations of angiosperm plants in a forested landscape or biome and their associations, the part of species in the percentage of floristic life-forms that resulted by the existing ecological parameters and environmental gradients. Life-form analysis provides a clear image of the sacred grove's biological spectrum. In the present study therophytes, chamaephytes and cryptophyte share the importance depicting the "thero-chamae-cryptophytic" phytoclimate. It may also be noted that the information acquired from this study will in future serve as a database of life forms for research of change detection and tenacity of bioclimate or phytoclimate (Raju et al., 2014). Comparing and contrasting the neighboring natural strands pattern along the environmental gradients will also be useful, revealing more than the mere forest covers in the ecosystem data; suggesting that biotic variables play a significant part in shaping a landscape's vegetation by guiding succession. This shows the impact in the 
sacred grove of anthropogenic disturbances that favor the development of more therophytes. Further disruption to the current sacred grove can therefore promote future changes in its current phytoclimate.

\section{Acknowledgement}

The authors are indebted to the informants and local tribal communities for cooperating and sharing their knowledge. Without their contribution, this study would have been impossible.

\section{References}

Anderson, T., 1862. Catalogue of plants indigenous in the neighbourhood of Calcutta with directions for examination and preservation of plants, Calcutta, India.

Anon, 2011. District Human Development Report, Paschim Medinipur, Government of West Bengal, Development and Planning Department, 306.

Badshah, L., Hussain, F. and Sher, Z., 2016. Floristic inventory, eco-logical characteristics and biological spectrum of plants of Parachinar, Kurram agency, Pakistan. Pakistan Journal of Botany, 48:1547-1558.

Batalha, M.A. and Martins, F.R., 2004. Floristic, frequency, and vegetation life-form spectra of a cerrado site. Brazilian Journal of Biology, 64:201-209.

Bennet, S.S.R., 1979. Flora of Howrah district, Dehradun, India, International Book Distributors, 406.

Berkes, F., 2009. Community conserved areas: policy issues in historic and contemporary context. Conservation Letters, 2:20-25.

Bohman K., 2004. Functional and morphological diversity of trees in different land use types along a rainforest margin in Sulawesi. [Ph.D. Thesis] Indonesia, Gottingen, University of Gottingen.

Brummitt, N., Bachman, S.P. and Moat, J., 2008. Applications of the IUCN Red List: towards a global barometer for plant diversity. Endanger Species Research, 6:127-135.

Cain, S.A. and Castro, G.D.O., 1959. Manual of vegetation analysis, New York, USA, Harper and Brothers, 325.

Cain, S.A., 1950. Life-form and phytoclimates. Botanical Review, 16:1-32.

Chase, M.W., Christenhusz, M.J.M., Fay, M.F., Byng, J.W., Judd, W.S., Soltis, D.E., Mabberley, D.J., Sennikov, A.N., Soltis, P.S. and Stevens P.F., 2016. An update of the angiosperm phylogeny group classification for the orders and families of flowering plants: APG IV. Botanical Journal of Linnaean Society, 181:1-20.

Chigani, H.K., Javadi, S.A., Zahedi-Amiri, G., Khajeddin, S.J. and Jafari, M., 2017. Vegetation composition differentiation and species-environment relationships in the northern part of Isfahan Province. Land Degradation and Development, 9:161-175.

Colding, J. and Folke, C., 1997. The relations among threatened species, their protection, and taboos. Conservation Ecology, 1:6.

Colding, J. and Folke, C., 2001. Social taboos: "invisible" systems of local resource management and biological conservation. Ecological Applications, 11:584-600.

Cunningham, S.A., Summerhayes, B. and Westoby, M., 1999. Evolutionary divergences in leaf structure and chemistry, comparing rainfall and soil nutrient gradients. Ecological Monographs, 69:569588.

Datta, S.C. and Majumdar, N.C., 1966. Flora of calcutta and vicinity. Bulletin of Botanical Society of Bengal, 20:16-120.

Ghildiyal, J.C., Barthwal, N. and Khantwal, A., 2016. Life-forms and biological spectrum of Deeva Ka Daanda a sacred grove in Garhwal Himalaya. Indian Forester, 142:167-178.

Gillison, A.N., 2018. Latitudinal variation in plant functional types. In: Geographical Changes in Vegetation and Plant Functional Types, Springer, Cham, pp. 21-57. 
Gnanasekaran, G., Nehru, P. and Narasimhan, D., 2012. Angiosperms of Sendirakillai sacred grove (SSG), Cuddalore district, Tamil Nadu, India. Check List, 8:113-129.

Gokhale, Y., Pala, N.A., Negi, A.K., Bhat, J.A. and Todaria, N.P., 2011. Sacred landscapes as repositories of biodiversity: A case study from the Hariyali Devi sacred landscape, Uttarakhand. International Journal of Conservation Science, 2:37-44.

Haines, H.H., 1921-1925. The Botany of Bihar and Orissa, Vol 1-6 (Reprint 1961), Calcutta, India, Botanical Survey of India.

Hooker, J.D., 1872-1897. The Flora of British India, Vols. 1-7 (Repr. 1973), Dehra Dun, India, Bishen Singh Mahendra Pal Singh.

Hughes, J.D. and Chandran, M.D.S., 1998. Sacred groves around the earth: An overview. In: Ramakrishnan, P.S., Saxena, K.G., Chandrashekara, U.M. (Eds.), Conserving the Sacred for Biodiversity Management. Oxford and IBH Publ. Co. Pvt. Ltd., New Delhi, India, pp. 69-86.

IUCN, 2020. The IUCN Red List of Threatened Species. Version 2020-1, https:/www.iucnredlist.org. Accessed 29 June 2020.

Jain S.K. and Rao, R.R., 1977. A Handbook of field and herbarium methods, Today and Tomorrow's Printers and Publishers, New Delhi, India, 157.

Meher-Homji, V.M., 1964. Life forms and biological spectra as epharmonic criteria of aridity and humidity in tropics. Journal of Indian Botanical Society, 43:424-430.

Mendez, E., 2005. The vegetation of the Laguna de Llancanelo provincial reserve (Mendiza, Argentina). Candollea, 60:123-148.

Misra, M.P., Mishra, P.N. and Pandey, B.N., 1979. An ecological account of the vegetation of Brahmyoni Hills (Gaya). Proceedings of the Indian Science Congress, 3:108-109.

Mitra J.N., 1958. Flowering plants of eastern India, Vol. 01. Monocotyledons, The World Press Private Ltd., Calcutta, 388.

Muller D. and Ellenberg, H., 1974. Aims and methods of vegetation ecology, John Wiley and Sons, New York, USA, p. 547.

Prain D., 1903. Bengal plants, Vol. 01 and 02, Botanical Survey of India, Calcutta, India, p. 670.

Rajendraprasad, M., Krishnan, P.N. and Pushpangadan, P., 1998. The life form spectrum of sacred grove and a functional tool to analyse the vegetation. Tropical Ecology, 39:211-217.

Raju, V.S., Krishna, P.G. and Suthari, S., 2014. Environmental assessment of climate of a habitat through floristic life-form spectra, a case study of Warangal north forest division, Telangana, India. Journal of Natural Sciences, 2:77-93.

Rana, T.S., Datt, B. and Rao, R.R., 2002. Life forms and biological spectrum of the flora of Tons Valley, Garhwal Himalaya (Uttaranchal), India. Taiwania, 47:164-169.

Raunkiaer C., 1934. The life forms of plants and statistical plant geography, Clarendon Press, Oxford, p. 632.

Reddy, S.C., Krishna, H.P., Meena, S.L., Bhardwaj, R. and Sharma, K.C., 2011. Composition of life forms and biological spectrum along climatic gradient in Rajasthan, India. International Journal of Environmental Science, 7:1632-1639.

Sanyal, M.N., 1994. Flora of Bankura district, Bishen Singh Mahendra Pal Singh, Dehra Dun, India, p. 555.

Saxena, A.K., Pandey, P. and Singh, J.S., 1982. Biological Spectrum and other structural functional attributes of the vegetation of Kumaun Himalaya. Plant Ecology, 49:111-119.

Sen, U.K. and Bhakat, R.K., 2009. Ecological role of sacred groves in plant conservation. Effects of environmental toxicology on human health, National Environmental Science Academy, New Delhi, India, pp. 211-219. 
Sen, U.K. and Bhakat, R.K., 2012. Sacred grove and plant conservation: An insight from taxonomy and ecology. In: Maiti, G.G., Mukherjee, S.K. (Eds.), Mutidisciplinary Approaches in Angiosperm Systamatics. Department of Botany, University of Kalyani, West Bengal, India, pp. 410-421.

Sen, U.K. and Bhakat, R.K., 2018. In situ conservation of a sacred grove in West Midnapore district, West Bengal, India: an integrated socio-ecological approach. Journal of Economic and Taxonomic Botany, 42:49-64.

Sen, U.K. and Bhakat, R.K., 2019a. Floristic and Phytoclimatic study of a sacred grove vegetation of West Midnapore district, West Bengal, India. Journal of Tropical Life Science, 9:119-138.

Sen, U.K. and Bhakat, R.K., 2019b. Assessment of the floristic composition, biological spectrum, leaf size spectra and traditional conservation management of a sacred grove in West Midnapore District, West Bengal, India. Indian Forester, 145:156-171.

Sen, U.K. and Bhakat, R.K., 2019c. Floristic composition, biological spectrum and conservation status of a sacred grove from Jhargram district, West Bengal, India. Indian Journal of Forestry, 42:161172.

Sen, U.K., 2018. Assessing the social, ecological and economic impact on conservation activities within human-modified landscapes: A case study in Jhargram District of West Bengal, India. International Journal of Conservation Science, 9:319-336.

Sen. U.K., 2016. Botanical and socio-cultural studies on some sacred groves of West Midnapore district, West Bengal, Ph.D. Thesis, Midnapore, India, Vidyasagar University, p. 321.

Sher, Z., Hussain, F. and Badshah, L., 2014. Biodiversity and Ecological characterization of the flora of Gadoon Rangeland, district Swabi, Khyber Pukhtunkhwa, Pakistan. Iranian Journal of Botany, 20:96-108.

Singh, R.I. and Gupta, A., 2015. Life-form classification and biological spectrum of Amambilok sacred grove, Andro, Manipur in northeast India. Pleione, 9:356-364. 